\title{
A systematic review of the psychometric properties of the cross-cultural translations and adaptations of the Multidimensional Perceived Social Support Scale (MSPSS)
}

\author{
Jermaine M. Dambi ${ }^{1,2^{*}} \mathbb{D}$, Lieselotte Corten ${ }^{1} \mathbb{D}$, Matthew Chiwaridzo ${ }^{1,2} \mathbb{D}$, Helen Jack ${ }^{3,4} \mathbb{D}$, Tecla Mlambo ${ }^{2} \mathbb{E}$
} and Jennifer Jelsma ${ }^{1}$ (D)

\begin{abstract}
Background: Social support (SS) has been identified as an essential buffer to stressful life events. Consequently, there has been a surge in the evaluation of SS as a wellbeing indicator. The Multidimensional Perceived Social Support Scale (MSPSS) has evolved as one of the most extensively translated and validated social support outcome measures. Due to linguistic and cultural differences, there is need to test the psychometrics of the adapted versions. However, there is a paucity of systematic evidence of the psychometrics of adapted and translated versions of the MSPSS across settings.
\end{abstract}

Objectives: To understand the psychometric properties of the MSPSS for non-English speaking populations by conducting a systematic review of studies that examine the psychometric properties of non-English versions of the MSPSS.

Methods: We searched Africa-Wide Information, CINAHL, Medline and PsycINFO, for articles published in English on the translation and or validation of the MSPSS. Methodological quality and quality of psychometric properties of the retrieved translations were assessed using the COSMIN checklist and a validated quality assessment criterion, respectively. The two assessments were combined to produce the best level of evidence per language/translation.

Results: Seventy articles evaluating the MSPSS in 22 languages were retrieved. Most translations [16/22] were not rigorously translated (only solitary backward-forward translations were performed, reconciliation was poorly described, or were not pretested). There was poor evidence for structural validity, as confirmatory factor analysis was performed in only nine studies. Internal consistency was reported in all studies. Most attained a Cronbach's alpha of at least 0.70 against a backdrop of fair methodological quality. There was poor evidence for construct validity.

Conclusion: There is limited evidence supporting the psychometric robustness of the translated versions of the MSPSS, and given the variability, the individual psychometrics of a translation must be considered prior to use. Responsiveness, measurement error and cut-off values should also be assessed to increase the clinical utility and psychometric robustness of the translated versions of the MSPSS.

Trial registration: PROSPERO - CRD42016052394.

Keywords: Multidimensional perceived social support, Translation, Adaptation, Validation, Reliability, Validity

\footnotetext{
* Correspondence: jermainedambi@gmail.com; dmbjer001@myuct.ac.za

${ }^{1}$ Division of Physiotherapy, School of Health and Rehabilitation Sciences,

University of Cape Town, Observatory, Cape Town, South Africa

${ }^{2}$ Rehabilitation Department, College of Health Sciences, University of

Zimbabwe, P.O Box A178, Avondale, Harare, Zimbabwe

Full list of author information is available at the end of the article
}

(c) The Author(s). 2018 Open Access This article is distributed under the terms of the Creative Commons Attribution 4.0 International License (http://creativecommons.org/licenses/by/4.0/), which permits unrestricted use, distribution, and reproduction in any medium, provided you give appropriate credit to the original author(s) and the source, provide a link to the Creative Commons license, and indicate if changes were made. The Creative Commons Public Domain Dedication waiver (http://creativecommons.org/publicdomain/zero/1.0/) applies to the data made available in this article, unless otherwise stated. 


\section{Background}

Social support (SS) is an essential buffer to stressful life events [1-13]. An adequate amount of SS improves mental health by mitigating the effects of negative psychosocial outcomes such as depression, anxiety, low self-efficacy, stress and loneliness or social isolation [1, 3, 4, 6, 9, 14-16]. Further, SS is a multidimensional, latent variable that dependents upon an individual's politico-social environment, socialization process and personal values/ethos amongst other factors $[1,3,10-12,17,18]$. The conceptualization and perception of SS is both complex and diverse, as testified by a plethora of conceptual frameworks and definitions which have been postulated to describe this subjective and yet important phenomenon [12, 13, 15, 16]. Social support can be defined as the amount of assistance one gets through interactions with other people $[15,16]$. The support can be either emotional (e.g. empathy), tangible (e.g. practical help) or informational (e.g. advice) $[15,16,19,20]$.

Various outcome measures have been developed to measure SS $[3,11]$. Originally created to measure SS in American adolescents, the Multidimensional Scale of Perceived Social Support (MSPSS) has evolved as one of the most extensively used SS outcome measure [3, 6-8, 11, 12, 18]. The MSPSS has 12 items that measure the perceived adequacy of the available amount of SS [15, 21, 22] (See Additional file 1). It measures the amount of SS an individual receives from three sources i.e. friends, family and significant other/special person. The amount of SS is rated on a seven-point Likert scale; with responses ranging from very strongly disagree $(=1)$ to very strongly agree (=7). The cumulative/total scores ranges from 12 to 84. As no item response theory calibration has been applied to the tool, the scores are interpreted as, the higher the score, the greater the amount of available SS [21]. The original version of the MSPSS yielded a three-factor structure, high internal consistency $(\alpha=0$. 88), stability (yielded $\alpha=0.85$ after 3 months from first administration) and moderate construct validity as the SS scores were negatively correlated to anxiety $(r=-0.18 ; p<0.01)$ and depression scores $(r=-24$; $p<0.01)$ [22].

The drive towards evidence-based practise (EBP) and patient-centred care has led to an increase in the crosscultural adaptation and translation of patient-reported outcome measures (PROMs) [7, 23-25]. To this end, the MSPSS has undergone extensive translation and adaption across linguistic and socio-economic contexts and settings i.e. from low- $[7,13,26]$ and middle $[11,18,27-29]$ to high-income countries [3, 6, 9, 17, 18, 30-34]. However, evidence of the psychometric properties of the translated and adapted versions of the MSPSS is fragmented, but deeply important to both clinical practice and research. For instance, due to conceptual differences, some authors have collapsed the scoring system (response options) to three [30, 35], four [12], five [13, 26, 36], and six [31] levels against the original seven-point Likert scoring system. However, the category "reordering" was based on "qualitative" analysis yet in those circumstances, item response theory techniques such as Rasch analysis are a prerequisite for ensuring the interpretability of response categories for translated tools $[37,38]$. Secondly, due to linguistic differences, some of the terminology of the original MSPSS have been changed, for example, the term "someone special" has been replaced by the term "husband" for some translations [5]. Thirdly, cultural differences are likely to influence perception of social support $[10-12,17,18]$ thus potentially influencing the structural validity of the translated versions of the MSPSS. For example, single factor structures were reproduced in the validation of the MSPSS in Asian countries such as Turkey [39-42], Thailand [28, 43, 44] and Pakistan [45-49], which are considered as "collectivistic" societies [50]. This implies that respondents could not differentiate between support provided by family, friends and significant others as postulated by the developers. The MSPSS was originally validated in the US which is considered an "individualistic" society [51]. Given the wide variation in MSPSS translations and cultural adaptations, a systematic evaluation of the adapted and translated versions of the MSPSS will help bring an understanding of the quality of the existing tools, and gaps in knowledge and adaptation [7, 23, 24, 52]. Further, a recent literature review asserts the psychometric robustness of the MSPSS across various settings and study populations [53]. However, the methodologies of the cited studies were not critically appraised. This is a limitation as the reported psychometrics are dependent on the quality of the methodologies applied in accordance with the COnsensus-based Standards for the selection of health status Measurement Instruments (COSMIN) criterion [54-57] . For instance, issues such as the sampling, handling of missing responses, the model used for analysis, bias in research reporting amongst other factors affects both the internal and external validity of the psychometric evaluation studies $[55,56,58]$. More so, other psychometric properties such as floor and ceiling effects, critical/cut off values and responsiveness were not reported and this weakens the level of evidence of the purported psychometric robustness of the MSPSS [53]. Therefore, the aims of the present review are to: (I) systematically identify the translated and adapted versions of the MSPSS; (II) evaluate the methodologies applied in the adaptation and translation process; and (III) appraise the psychometric properties of the translated and adapted versions.

\section{Methods}

\section{Protocol and registration}

This review was conducted in accordance with the Preferred Reporting Items of Systematic Reviews and 
Meta-Analyses Protocol (PRISMA) guidelines (Additional file 2). The protocol was registered with and published on the PROSPERO database (Ref-CRD42016052394) [59].

\section{Eligibility criteria}

Studies were included if one of the aim(s) was to: translate and culturally adapt the MSPSS; evaluate the psychometrical properties of translated version(s) of the MSPSS or if they measured SS using translated versions of the MSPSS. Additionally, only studies applying a quantitative study designs and reporting on the psychometrics of the translated, 12-item version of the MSPSS were included. Due to limitation in resources for translation, only full text articles published in English were included. Studies based in more than one country that applied the MSPSS as an outcome measure, commentaries, review articles and studies which utilized several linguistic versions of the MSPSS were excluded. Additionally, studies which utilized both the original version of the MSPSS and the translated version were also excluded as the aim of the review was to appraise the psychometric properties of the translated versions of the MSPSS.

\section{Information sources}

We searched Africa-Wide information, CINAHL, PubMed, Psych INFO, and Scopus for peer-reviewed articles. Google Scholar was also searched to identify grey literature such as dissertations. We also contacted the developer of the MSPSS to identify the translations which we might have missed through database searches. We did not impose a time limit to publication dates to gather as many articles as possible. In cases where the abstract was available online and where it was unclear as to whether the translated version of the MSPSS was applied, the authors were contacted for clarification. Reference lists of the identified articles were manually searched for possible articles for inclusion for the attainment of literature saturation. Literature searches were conducted between November 2016 and February 2017.

\section{Search}

Outlined in Table 1 below is the search strategy applied in retrieving articles on EBSCO-host search engine:

As an illustration, articles on the translation and adaptation of the French version of the MSPSS were retrieved as follows: \{multidimensional scale of perceived social support OR MSPSS OR mspss\} AND \{translation OR translated OR translat" \} AND \{france OR France OR French OR french OR francias\}.

\section{Study selection}

A previously described study selection process was utilized [60, 61]. One author (JD) ran the search strategy
Table 1 Search strategy

\begin{tabular}{ll}
\hline Key term & Alternative term(s) \\
\hline $\begin{array}{l}\text { multidimensional scale of perceived } \\
\text { social support }\end{array}$ & MSPSS, mspss \\
translation & translated, translat* \\
adaption & Cultural adaptation, adapt* \\
- Language in which the MSPSS was & \\
translated to e.g. French & \\
- Country where the translation was & \\
$\quad$ done e.g. France & \\
\hline
\end{tabular}

across all databases. Two independent reviewers (MC and LC) then selected the eligible titles and abstracts for further investigation using a predefined search strategy. Reviewers resolved disagreements about inclusion through discussion, and a, third reviewer (JD) was brought in if the two reviewers could not agree. Another author (MC) manually searched the reference lists of identified articles to screen full texts for inclusion.

\section{Data collection process}

The principal investigator (JD) developed the data collection sheet. The tool/checklist was validated by three co-investigators (MC, TM \& JD) with prior experience with psychometrics and psychometrics systematic reviews. The tool was then piloted on ten randomly selected studies of definite rating. Three reviewers (MC, JD \& TM) independently applied the COSMIN checklist to rate the methodological quality of the ten studies. The inter-rater reliability was 0.8 as measured by the Kappa coefficient. Most of the disparities emanated from the rating of the structural validity and differences were discussed upon further reference to the COSMIN user manual. Afterwards, two reviewers (MC \& TM) independently extracted data from the retrieved studies and their inter-rater reliability was 0.9 . The two data collection sheets were reconciled into one data set through discussions between the principal author (JD) and two reviewers (MC \& TM).

\section{Data items}

The extracted information included the research setting and design, study sample, demographic and or clinical characteristics of the participants, target translation language and secondary outcome measures e.g. depression. The methodological quality of the translation process and evidence for reliability and validity of the questionnaires was also documented.

\section{Risk of bias in individual studies}

The methodological quality of the retrieved articles was assessed using the COnsensus-based Standards for the selection of health status Measurement Instruments 
(COSMIN) checklist [54]. It consists of eight boxes which evaluate the methodological quality of the following psychometrics: internal consistency (box A), reliability (box B), measurement error (box C), content validity (box D), structural validity (box E), hypotheses testing (box F), cross-cultural validity (box G), and criterion validity (box H) [54, 55]. Methodological quality is rated on a four-point Likert scale i.e. poor, fair, good and excellent. In assessing a domain, the lowest rating of an item is assigned as the overall quality of the domain under investigation. For example, in assessing structural validity, if an inappropriate rotation method is employed i.e. if orthogonal rotation instead of oblique rotation is used to interpret factors on the MSPSS, the evidence will be rated as poor even if all the other sub-domains are rated as of excellent quality [54-57].

\section{Best evidence synthesis}

To come up with best evidence synthesis for each psychometric property, we used the Cochrane Collaboration Back Review Group criterion [62] as outlined in Table 2 to synthesise results from the COSMIN methodological assessment [54-57] (see Table 3) and Terwee et al. criterion for evaluation of quality of psychometrics [58] (see Table 4).

\section{Results \\ Study selection \\ Study characteristics}

Description of study participants and settings $A$ total of 22 translations were retrieved from 70 studies. A sample of convenience was the most common method of participant selection $[n=7,31.8 \%]$, and translations were most often validated using a cross sectional study design [72.7\%, $n=16]$. Most studies were from high-income settings [72.7\%, $n=16]$ and conducted in clinical settings $[45.8 \%, n=11]$ or at universities [29.1\%, $n=7]$. Participants were of varying ages with the youngest and eldest groups averaging 14.8 (SD 1.6) and 58.7 (SD 13.2) years respectively Table 5.

Description of adaptations For seven of the translations, the response options were reduced from the original seven -point Likert scale to a five $(n=4)$, three $(n=1)$, four $(n=1)$ and six-point $(n=1)$ scale. Some of the original terms on

Table 2 Best evidence synthesis of the psychometric properties

\begin{tabular}{|c|c|c|c|c|c|c|}
\hline Version -Country & $\begin{array}{l}\text { Internal } \\
\text { consistency }\end{array}$ & $\begin{array}{l}\text { Criterion } \\
\text { validity }\end{array}$ & $\begin{array}{l}\text { Construct validity - } \\
\text { convergent }\end{array}$ & $\begin{array}{l}\text { Construct validity- } \\
\text { divergent }\end{array}$ & $\begin{array}{l}\text { Reproducibility- } \\
\text { agreement }\end{array}$ & $\begin{array}{l}\text { Reproducibility- } \\
\text { reliability }\end{array}$ \\
\hline Arabic women (MSPSS-AW)- USA & Moderate (--) & & Limited (-) & Limited (-) & & \\
\hline Arabic Generic - Lebanon & Limited (-) & & Unknown (?) & Unknown (?) & & \\
\hline Chichewa- Malawi & Strong $(+++)$ & & & Limited (-) & & \\
\hline Chinese (Simplified) - Malaysia & Strong $(+++)$ & Unknown (?) & & Unknown (?) & & Unknown (?) \\
\hline Chinese (Traditional) - Hong Kong, China & Conflicting (-) & Unknown (?) & Moderate (--) & Moderate (--) & & Limited (+) \\
\hline Chiyao - Malawi & Strong $(+++)$ & & & Limited (-) & & \\
\hline Creole- USA (Haiti) & Unknown (?) & & Unknown (?) & & & Unknown (?) \\
\hline French - France & Limited (+) & & & Limited (-) & & Limited (-) \\
\hline Hausa - Nigeria & Strong $(+++)$ & & & Limited (-) & & Limited (-) \\
\hline Korean-Korea & Strong $(+++)$ & & Limited (-) & Limited (-) & & \\
\hline Luganda - Uganda & Limited (-) & & & & & \\
\hline Malay - Malaysia & Unknown (?) & Unknown (?) & & Unknown (?) & & Unknown (?) \\
\hline Persian - Iran & Limited (-) & & Unknown (?) & & & Limited (-) \\
\hline Polish - Poland & Strong $(+++)$ & & Moderate (--) & Moderate (--) & & \\
\hline Portuguese - Portugal & Strong $(+++)$ & & Limited (-) & Limited (+) & Limited (-) & \\
\hline Spanish - *USA, ** Spain & Limited (+) & Moderate (--) & Moderate (--) & & & \\
\hline Swedish - Sweden & Moderate $(++)$ & & Limited (-) & & & Limited (+) \\
\hline Tamil - Malaysia & Limited (-) & Unknown (?) & Unknown (?) & Unknown (?) & & \\
\hline Thai - Thailand & Moderate $(++)$ & & Unknown (?) & & & Limited (+) \\
\hline Turkish (Original) - Turkey & Moderate $(++)$ & & Conflicting $(-)$ & Conflicting (+) & & \\
\hline Turkish (Revised) - Turkey & Conflicting (-) & & Unknown (?) & Unknown (?) & & Unknown (?) \\
\hline Urdu - Pakistan & ? (unknown) & & Limited (-) & Moderate (--) & & \\
\hline
\end{tabular}

* and ${ }^{* *}$ denotes findings from the USA and Spain respectively 
Table 3 Methodological ratings of retrieved studies

\begin{tabular}{|c|c|c|c|c|c|c|c|}
\hline \multirow[t]{2}{*}{ Version -Country } & \multirow{2}{*}{$\begin{array}{l}\text { Crosscultural } \\
\text { validity }\end{array}$} & \multirow{2}{*}{$\begin{array}{l}\text { Structural } \\
\text { validity }\end{array}$} & \multirow{2}{*}{$\begin{array}{l}\text { Internal } \\
\text { consistency }\end{array}$} & \multirow[t]{2}{*}{ Reliability } & \multicolumn{2}{|c|}{ Hypothesis testing/construct validity } & \multirow{2}{*}{$\begin{array}{l}\text { Criterion } \\
\text { validity }\end{array}$} \\
\hline & & & & & Divergent validity & Convergent validity & \\
\hline Arabic women - USA & Poor [5] & Fair [5] & Fair [5] & Fair & {$[5,47]$} & Fair [5] & \\
\hline Arabic Generic - Lebanon & Poor [27] & Poor [27] & Fair [27] & Fair [27] & Fair [27] & & \\
\hline Chichewa- Malawi & Fair [26] & Excellent [26] & Excellent [26] & Fair $[26,36]]$ & & & \\
\hline Chinese (Simplified) - Malaysia & Poor [12] & Poor [12] & Fair [12] & Poor [12] & Poor [12] & & Poor [12] \\
\hline $\begin{array}{l}\text { Chinese (Traditional) } \\
- \text { Hong Kong, China }\end{array}$ & Poor [17] & $\begin{array}{l}\text { Poor }[17] \\
\text { Fair }[64,65]\end{array}$ & $\begin{array}{l}\text { Excellent }[17] \\
\text { Fair }[64,80]\end{array}$ & Fair [64] & $\begin{array}{l}\text { Poor [17], }[73,76] \\
\text { Fair }[64,75-78,80-84]\end{array}$ & $\begin{array}{l}\text { Poor }[73,84] \\
\text { Fair }[74,76,78-82,84]\end{array}$ & Fair [17] \\
\hline Chiyao - Malawi & Fair [26] & Excellent [26] & Excellent [26] & Fair [26] & & & \\
\hline Creole- Haiti(USA) & Poor [6] & & Poor [6] & Fair [6] & Fair [6] & & \\
\hline French - France & Poor [9] & Fair [9] & Fair [9] & Fair [9] & Fair [9] & & \\
\hline Hausa - Nigeria & Good $[7,14]$ & Excellent [14] & Excellent [14] & Fair [14] & Fair [85] & & \\
\hline Korean-Korea & Poor [87] & Poor [87] & Excellent [87] & Fair [87] & Fair [87] & & \\
\hline Luganda - Uganda & Fair [13] & Poor [13] & Fair [13] & & & & \\
\hline Malay - Malaysia & Poor [11] & Poor $[11,88]$ & Fair $[11,88]$ & Poor [11] & $\begin{array}{l}\text { Poor }[11,88] \text { : } \\
\text { Fair }[89]\end{array}$ & Poor [11] & Poor [11] \\
\hline Persian - Iran & Poor [18] & Poor [18] & Fair [18] & Fair [18] & Poor $[90,91]$ & & \\
\hline Polish - Poland & Good [92] & Excellent [92] & Excellent [92] & & $\begin{array}{l}\text { Fair }[92,93] \\
\text { Good }[94]\end{array}$ & $\begin{array}{l}\text { Fair }[79,92,93] \\
\text { Good }[94]\end{array}$ & \\
\hline Portuguese - Portugal & Fair [32] & Excellent [32] & Excellent [32] & Fair [32] & $\begin{array}{l}\text { Fair [96, 97], } \\
\text { Good [32] }\end{array}$ & Fair [96] & \\
\hline Spanish - *USA, ${ }^{*}$ Spain & Poor ** [99] & $\begin{array}{l}\text { Poor * [101], } \\
\text { Fair ** [99] }\end{array}$ & Fair * [101] & & Fair ** $[34,98,99]$ & Fair ** $[34,98,99]$ & \\
\hline Swedish - Sweden & Good [3] & Poor [3] & Good [3] & Fair [3] & & $\begin{array}{l}\text { Good [3], } \\
\text { Fair [102] }\end{array}$ & \\
\hline Tamil - Malaysia & Poor [87] & Poor [87] & Fair [87] & & Poor [87] & Poor [87] & Poor [87] \\
\hline Thai - Thailand & Poor [44] & Good $[43,44]$ & Good $[43,44]$ & Fair [44] & Fair $[28,43,44]$ & Fair $[28,43,44]$ & \\
\hline Turkish (Original)- Turkey & Poor [39] & Poor $[39,41]$ & Fair $[39,41]$ & & $\begin{array}{l}\text { Poor }[41,42], \\
\text { Fair }[39,40]\end{array}$ & $\begin{array}{l}\text { Poor }[41,42] \\
\text { Fair }[40]\end{array}$ & \\
\hline Turkish (Revised) - Turkey & & Fair $[29,104]$ & Fair [29] & Poor [29] & Fair [29] & Poor [29] & \\
\hline Urdu - Pakistan & Poor [49] & $\begin{array}{l}\text { Poor [49] } \\
\text { Fair [47] }\end{array}$ & Poor [49] & & Fair [45-48] & Fair [47] & \\
\hline
\end{tabular}

* and ${ }^{* *}$ denotes findings from the USA and Spain respectively

the MSPSS were modified/changed in four of the studies $i$. e. the term special person/significant other was changed to "husband" or "spouse". The MSPSS was self-administered in most studies [54.5\%, $n=12$ ]. Depression, general psychological well-being, social networks and anxiety were the most commonly measured secondary outcome measures Fig. 1 and Table 6.

\section{Results of individual studies}

\section{Arabic}

Two variants of Arabic translations were retrieved [27, 35].

\section{Arabic generic version}

The Arabic generic version was described in one study [27]. The methodology for this cross-cultural validation study was poor, as scanty details were provided for the adaptation process. The evidence for structural validity was poor as only exploratory factor analysis (EFA) was performed. There was limited evidence for internal consistency (IC) as the handling of missing responses was not reported. There was unknown evidence for construct validity as no specific hypotheses were formulated.

\section{Arabic version for women (MSPSS-AW)}

Two studies on the MSPSS-AW were available [35, 63]. The methodology for the cross-cultural validation was poor. There was no description of; translators' expertise, whether the translations were done independently, the number of forward and backward translations performed and the reconciliation process. This version was not reviewed by a committee and was not pretested. There was moderate evidence for $\mathrm{IC}$, the handling of missing responses was not reported. There was fair evidence for structural validity, although confirmatory factor analysis (CFA was performed, the number of missing responses 
Table 4 Ratings of quality of psychometric properties

\begin{tabular}{|c|c|c|c|c|c|c|}
\hline Version -Country & $\begin{array}{l}\text { Cross-cultural } \\
\text { validity }\end{array}$ & $\begin{array}{l}\text { Structural } \\
\text { validity }\end{array}$ & $\begin{array}{l}\text { Internal } \\
\text { consistency }\end{array}$ & Reliability & $\begin{array}{l}\text { Construct } \\
\text { validity }\end{array}$ & $\begin{array}{l}\text { Criterion } \\
\text { validity }\end{array}$ \\
\hline Arabic women - USA & $?[5]$ & $-[5]$ & $+[5]$ & & $?[63]$ & \\
\hline Arabic Generic - Lebanon & $?[27]$ & $?[27]$ & $+[27]$ & & $?[27]$ & \\
\hline Chichewa- Malawi & - [26] & $+[26]$ & $+[26]$ & & $?[26,36]$ & \\
\hline Chinese (Simplified) - Malaysia & $?[12]$ & $?[12]$ & $+[12]$ & $?[12]$ & $?[12]$ & $?[12]$ \\
\hline Chinese (Traditional) - Hong Kong, China & $?[17]$ & $?[17],-[64,80]$ & $?[17],+[64],-[80]$ & $?[17],+[64]$ & $?[17,64-84]$ & $?[17]$ \\
\hline Chiyao - Malawi & $-[26]$ & $+[26]$ & $+[26]$ & & $?[26,36]$ & \\
\hline Creole- Haiti(USA) & $?[6]$ & $?[6]$ & $?[6]$ & $?[6]$ & & \\
\hline French - France & ? [9] & - [9] & $+[9]$ & $?[9]$ & $?[9]$ & \\
\hline Hausa - Nigeria & $-[7,14]$ & $+[14]$ & $+[14]$ & $?[14]$ & $?[85]$ & \\
\hline Korean-Korea & $?[87]$ & $?[87]$ & $+[87]$ & & $?[87]$ & \\
\hline Luganda - Uganda & - [13] & $?[13]$ & $?[13]$ & & & \\
\hline Malay - Malaysia & $?[11]$ & $?[11,88]$ & - $[11,88]$ & $?[11]$ & $?[11,88],-[89]$ & $?[11]$ \\
\hline Persian - Iran & $?[18]$ & $?[18]$ & $?[18]$ & $?[18]$ & $?[90,91]$ & \\
\hline Polish - Poland & - [92] & $+[92]$ & $+[92]$ & & $?$ [92-94] & \\
\hline Portuguese - Portugal & - [32] & $+[32]$ & $+[32]$ & $?[32]$ & $?[32,96,97]$ & \\
\hline Spanish - *USA, ${ }^{* *}$ Spain & ? [99]** & $?[85] *,-[99] * *$ & $+[85]^{*},+[99]^{* *}$ & & $?[34,84,99]^{* *},+[98]$ & \\
\hline Swedish - Sweden & - [3] & $?[3]$ & $+[3]$ & $+[3]$ & $+[3] ?[102]$ & \\
\hline Tamil - Malaysia & $?$ [103] & $?[103]$ & $?[103]$ & & $?[103]$ & $?[103]$ \\
\hline Thai - Thailand & $?[44]$ & $-[43,44]$ & $+[43,44]$ & & $?[28,43,44]$ & \\
\hline Turkish (Original)- Turkey & $?[39]$ & $?[39,41]$ & $+[39,41]$ & & $?[39-42]$ & \\
\hline Turkish (Revised) - Turkey & & $-[29,104]$ & $+[29] ; ?[104]$ & & $?[29]$ & \\
\hline Urdu - Pakistan & $?[49]$ & $?[49],-[47]$ & $?[49]$ & & $?[45-48]$ & \\
\hline
\end{tabular}

* and ** denotes findings from the USA and Spain respectively

was not documented. There was limited evidence of construct validity as no specific hypotheses were formulated and the psychometrics of the comparator instruments were not adequately described.

\section{Chichewa and Chiyao}

Two studies were available $[26,36]$. The methodology for the cross-cultural validation was fair as only one forward and one backward translations were performed. Further, the expertise of the translators, pre-test sample and the reconciliation of the forward translation were poorly described. There was strong evidence for structural validity and IC. Both EFA and CFA were applied for structural validity evaluation. There was limited evidence for construct validity, no specific hypotheses were formulated.

\section{Chinese}

Two versions in simplified and traditional Chinese versions were retrieved $[12,17]$.

\section{Traditional Chinese}

Twenty-one studies, applying the traditional Chinese version of the MSPSS, were available [17, [64-84]. The methodology for the cross-cultural validation was poor. Solitary forward and backward translations were performed; the translators' expertise were not outlined and it was not clear whether the translations were done independently and if the tool was pretested in the target population. Two studies tested structural validity of the MSPSS-C in different populations [17, 64]. There was poor evidence for structural validity as only EFA was performed. There was conflicting evidence for IC. The methodological quality of one of the studies was questionable [17] with the second study yielding a Cronbach's alpha $<0.70$ despite fair methodological quality [65]. There was limited evidence for reliability as the test conditions and the stability of the re-test sample were not clearly outlined. There was moderate evidence for construct validity, no specific hypotheses were formulated. The evidence for criterion validity was unknown as the psychometrics of the "purported" gold standard measure was questionable.

\section{Simplified Chinese}

Only one study was available [12]. The methodology for the cross-cultural validation was poor. The credentials 
Table 5 Study descriptions

\begin{tabular}{|c|c|c|c|c|c|c|c|c|}
\hline $\begin{array}{l}\text { Authors } \\
\text { [Year of publication] }\end{array}$ & Language (s) & Study aim(s) & Design & $\begin{array}{l}\text { Country - } \\
\text { [income bracket] }\end{array}$ & Setting & Participants & Sampling & $\begin{array}{l}\text { Age in years- } \\
\text { Mean [SD] }\end{array}$ \\
\hline Aroian et al. [2010] & Arabic & $T \& P T$ & Cross-sectional & USA - HIC & Community & $\begin{array}{l}\text { Arab Muslim } \\
\text { immigrant } \\
\text { married woman, } \\
N=539\end{array}$ & Convenience & $40.2(6.5)$ \\
\hline Norries et al. [2011] & Arabic & PT & Cross-sectional & USA - HIC & Community & $\begin{array}{l}\text { Arab Muslim } \\
\text { immigrant } \\
\text { women, } N=519\end{array}$ & Convenience & $40.22(6.5)$ \\
\hline Merhi \& Kazarian [2012] & Arabic & $T \& P T$ & Cross-sectional & Lebanon - UMIC & Community & $\begin{array}{l}\text { Healthy adults, } \\
N=221\end{array}$ & Not stated & $34.0(11.7)$ \\
\hline Stewart et al. [2014] & $\begin{array}{l}\text { Chichewa \& } \\
\text { Chiyao }\end{array}$ & $T \& P T$ & Cross-sectional & Malawi - LIC & Clinical & $\begin{array}{l}\text { Women } \\
\text { attending } \\
\text { antenatal visits, } \\
N=583\end{array}$ & Convenience & $25.1(6.2)$ \\
\hline Stewart et al. [2014] & $\begin{array}{l}\text { Chichewa \& } \\
\text { Chiyao }\end{array}$ & PT & Cross-sectional & Malawi - LIC & Clinical & $\begin{array}{l}\text { Women attending } \\
\text { antenatal visits, } \\
N=583\end{array}$ & Consecutive & $25.14(6.22)$ \\
\hline Cao et al. [2015] & Chinese & PT & Cross sectional & China - HIC & Community & $\begin{array}{l}\text { Elderly population, } \\
N=928\end{array}$ & $\begin{array}{l}\text { Two-stage } \\
\text { stratified } \\
\text { cluster } \\
\text { sampling }\end{array}$ & *60-94 [Range] \\
\hline Chan et al. [2010] & Chinese & PT & $\begin{array}{l}\text { Random } \\
\text { Controlled Trial }\end{array}$ & China - HIC & Clinical & $\begin{array}{l}\text { Patients with chronic } \\
\text { obstructive pulmonary } \\
\text { disease, } N=206\end{array}$ & Random & $72.9(7.7)$ \\
\hline He et al. [2016] & Chinese & PT & Cross sectional & China - HIC & Clinical & $\begin{array}{l}\text { Burns patients, } \\
N=246\end{array}$ & Not stated & $25.77(2.14)$ \\
\hline Liu et al. [2015] & Chinese & PT & Cross sectional & China - HIC & Community & Adults, $N=1471$ & Not stated & $34.5(10.4)$ \\
\hline Meng-Yao et al. [2016] & Chinese & PT & Cross sectional & China - HIC & Clinical & $\begin{array}{l}\text { Patients with } \\
\text { bladder cancer, } \\
N=365\end{array}$ & Convenience & $63.76(11.45)$ \\
\hline Tan et al. [2016] & Chinese & PT & Cross sectional & China - HIC & High schools & $\begin{array}{l}\text { Adolescents, } \\
N=618\end{array}$ & Random & $16.29(2.58)$ \\
\hline Taylor-Piliae et al. [2005] & Chinese & PT & Quasi-experimental & USA-HIC & Community & $\begin{array}{l}\text { Chinese nationals } \\
\text { with cardio } \\
\text { vascular disease } \\
\text { risk factors, } N=38\end{array}$ & Convenience & $66(8.3)$ \\
\hline Wang et al. [2014] & Chinese & PT & Cross sectional & China - HIC & Clinical & $\begin{array}{l}\text { Patients with } \\
\text { depression, } \\
N=100\end{array}$ & Not stated & $41.36(15.55)$ \\
\hline Wang et al. [2015] & Chinese & PT & Longitudinal & China - HIC & Clinical & $\begin{array}{l}\text { Patients with } \\
\text { breast cancer, } \\
N=404\end{array}$ & Not stated & $47.64(7.66)$ \\
\hline Zeng et al. [2016] & Chinese & PT & Longitudinal & China - HIC & Clinical & $\begin{array}{l}\text { Patients mild } \\
\text { traumatic brain } \\
\text { injury, } N=219\end{array}$ & Convenience & $34.7(14.8)$ \\
\hline Zhang et at [2016] & Chinese & PT & $\begin{array}{l}\text { Random } \\
\text { Controlled Trial }\end{array}$ & China - HIC & Clinical & $\begin{array}{l}\text { Outpatients with } \\
\text { mild depression, } \\
N=62\end{array}$ & Random & $48.3(17.5)$ \\
\hline Zhou et al. [2015] & Chinese & PT & Cross-sectional & China - HIC & Clinical & $\begin{array}{l}\text { Patients on } \\
\text { methadone } \\
\text { maintenance } \\
\text { treatment, } \\
N=1212\end{array}$ & Not stated & $42.5(6.2)$ \\
\hline Zhu, Hu \& Efird [2012] & Chinese & PT & $\begin{array}{l}\text { Cross-sectional, } \\
\text { correlational }\end{array}$ & China - HIC & Community & $\begin{array}{l}\text { Elderly population, } \\
N=120\end{array}$ & Quasi-random & $71.42(7.18)$ \\
\hline Chan, Yu \& Li [2011] & Chinese & PT & Cross sectional & China - HIC & Clinical & $\begin{array}{l}\text { Peritoneal dialysis } \\
\text { patients, } N=141\end{array}$ & Random & $57(12)$ \\
\hline Cheng et al. [2004] & Chinese & PT & Cross-sectional & $\begin{array}{l}\text { Hong Kong - } \\
\text { HIC }\end{array}$ & High schools & $\begin{array}{l}\text { Adolescents, } \\
N=2105\end{array}$ & Not stated & $14.8(1.6)$ \\
\hline Sing \& Wong [2011] & Chinese & PT & Cross sectional & Hong Kong - HIC & College & $\begin{array}{l}\text { College students, } \\
N=529\end{array}$ & Not stated & $21.1(1.77)$ \\
\hline Kee-Lee Chou [2000] & Chinese & $T \& P T$ & Cross-sectional & Hong Kong - HIC & High schools & $\begin{array}{l}\text { Adolescents, } \\
N=410\end{array}$ & Random & $17.5(0.7)$ \\
\hline
\end{tabular}


Table 5 Study descriptions (Continued)

\begin{tabular}{|c|c|c|c|c|c|c|c|c|}
\hline $\begin{array}{l}\text { Authors } \\
\text { [Year of publication] }\end{array}$ & Language (s) & Study aim(s) & Design & $\begin{array}{l}\text { Country - } \\
\text { [income bracket] }\end{array}$ & Setting & Participants & Sampling & $\begin{array}{l}\text { Age in years- } \\
\text { Mean [SD] }\end{array}$ \\
\hline Liu et al. [2015] & Chinese & PT & Cross sectional & China - HIC & Clinical & $\begin{array}{l}\text { Patients with } \\
\text { haematological } \\
\text { malignancies, } \\
N=225\end{array}$ & Consecutive & ${ }^{*}$ 15-83 [Range] \\
\hline Wong et al. [2012] & Chinese & PT & $\begin{array}{l}\text { Comparative cross- } \\
\text { sectional survey }\end{array}$ & Hong Kong - HIC & $\begin{array}{l}\text { 1. Clinical } 2 \text {. } \\
\text { Community }\end{array}$ & $\begin{array}{l}\text { 1. Caregivers of } \\
\text { stroke, Parkinson's } \\
\text { disease, or } \\
\text { Alzheimer disease } \\
\text { patients, } n=552 \text {. } \\
\text { General population, } \\
n=61\end{array}$ & Not stated & $\begin{array}{l}\text { 1. caregivers- } \\
72(6.2) \\
\text { 2. General } \\
\text { population- } 72 \\
\text { (6.3) }\end{array}$ \\
\hline Liu et al. [2015] & Chinese & PT & Cross sectional & China - HIC & University & $\begin{array}{l}\text { University students, } \\
N=722\end{array}$ & & $19.68(1.12)$ \\
\hline Yeung et al. [2013] & Chinese & PT & Quasi-experimental & USA-HIC & Clinical & $\begin{array}{l}\text { Chinese Americans, } \\
N=14\end{array}$ & Convenience & $53(14)$ \\
\hline Yu Ling et al. [2015] & Chinese & PT & Cross sectional & China - HIC & High schools & $\begin{array}{l}\text { Adolescents, } \\
N=1654\end{array}$ & Random & $15.85(1.02)$ \\
\hline Hannan et al. [2016] & Creole & $T \& P T$ & Longitudinal & USA-HIC & University & $\begin{array}{l}\text { Haitian } \\
\text { post-partum } \\
\text { mothers, } N=85\end{array}$ & Convenience & $45.8(11.1)$ \\
\hline Denis et al. [2015] & French & $T \& P T$ & Cross-sectional & France-HIC & Clinical & $\begin{array}{l}\text { Post-partum } \\
\text { mothers, } N=148\end{array}$ & Not stated & $30.5(5.1)$ \\
\hline Hamza et al. [2012] & Hausa & $\mathrm{T}$ & Mixed methods & Nigeria -LMIC & Clinical & $\begin{array}{l}\text { Patients with } \\
\text { stroke, } N=10\end{array}$ & Random & $\begin{array}{l}51.5 \\
\text { (not provided) }\end{array}$ \\
\hline Mohammad et al. [2015] & Hausa & PT & Cross-sectional & Nigeria - LMIC & Clinical & $\begin{array}{l}\text { Patients with } \\
\text { stroke, } N=140\end{array}$ & Consecutive & $58.8(13.2)$ \\
\hline $\begin{array}{l}\text { Vincent-Onabajo et al. } \\
\text { [2015] }\end{array}$ & Hausa & PT & Cross-sectional & Nigeria - LMIC & Clinical & $\begin{array}{l}\text { Patients with } \\
\text { stroke, } N=100\end{array}$ & Consecutive & $51.4(13.5)$ \\
\hline Park et al. [2011] & Korean & $T \& P T$ & Cross-sectional & Korea-HIC & Clinical & $\begin{array}{l}\text { Women with } \\
\text { diabetes, } N=123\end{array}$ & Convenience & $53.4(5.9)$ \\
\hline Nakigudde et al. (2009) & Luganda & $T \& P T$ & Cross-sectional & Uganda- LIC & Clinical & $\begin{array}{l}\text { Post-partum } \\
\text { mothers, } N=240\end{array}$ & Systematic & $26(5.7)$ \\
\hline $\mathrm{Ng}^{*}$ et al. [2010] & Malay & $T \& P T$ & Longitudinal & Malaysia-UMIC & Clinical & $\begin{array}{l}\text { University } \\
\text { students, } N=237\end{array}$ & Not stated & $\begin{array}{l}* 19-25 \\
\text { [range] }\end{array}$ \\
\hline $\mathrm{Ng}^{*}$ et al. [2015] & Malay & PT & Prospective cohort & Malaysia-UMIC & Clinical & $\begin{array}{l}\text { Female patients } \\
\text { with breast } \\
\text { cancer, } N=221\end{array}$ & Not stated & $551(11.5)$ \\
\hline Razali \& Yusoff [2014] & Malay & PT & Cross-sectional & Malaysia-UMIC & Clinical & $\begin{array}{l}\text { Patients with } \\
\text { Schizophrenia, } \\
N=70\end{array}$ & Universal & $33(9)$ \\
\hline Roohafza et al. [2016] & Persian & PT & Cross sectional & Iran-UMIC & Clinical & $\begin{array}{l}\text { Patients with } \\
\text { irritable bowel } \\
\text { syndrome, } \\
N=4763\end{array}$ & Not stated & Not stated \\
\hline $\begin{array}{l}\text { Bagherian-Sararoudi, } \\
\text { et al. [2013] }\end{array}$ & Persian & $\mathrm{T} \& \mathrm{PT}$ & Longitudinal & Iran-UMIC & Clinical & $\begin{array}{l}\text { 1.Myocardial } \\
\text { patients, } n=176 ; 2 \text {. Healthy } \\
\text { participants, } \\
n=71: N=247\end{array}$ & Not stated & 1. $56(9.8)$ \\
\hline Ghorbani et al. [2005] & Persian & PT & Cross sectional & Iran-UMIC & Clinical & $\begin{array}{l}\text { Parents of } \\
\text { pre-term \& } \\
\text { full-term infants, } \\
N=164\end{array}$ & $\begin{array}{l}\text { Multi-stage } \\
\text { sampling }\end{array}$ & $\begin{array}{l}\text { 1. pre-term - } \\
27.6(6.25) \\
\text { 2. full term- } \\
28.22(4.54)\end{array}$ \\
\hline $\begin{array}{l}\text { Adamczyk \& } \\
\text { DiTommaso [2014] }\end{array}$ & Polish & PT & Cross-sectional & Poland - HIC & University & $\begin{array}{l}\text { Young adults, } \\
N=417\end{array}$ & Not stated & $21.14(2.05)$ \\
\hline $\begin{array}{l}\text { Adamczyk \& } \\
\text { Segrin[2015] }\end{array}$ & Polish & PT & Cross-sectional & Poland - HIC & University & $\begin{array}{l}\text { Young adults, } \\
N=553\end{array}$ & Not stated & $23.42(3.27)$ \\
\hline Adamczyk [2013] & Polish & $\mathrm{T} \& \mathrm{PT}$ & Longitudinal & Poland - HIC & University & $\begin{array}{l}\text { University } \\
\text { students, } N=418\end{array}$ & Convenience & $21.1(2.1)$ \\
\hline $\begin{array}{l}\text { Adamczyk \& } \\
\text { Segrin[2015] }\end{array}$ & Polish & PT & Cross-sectional & Poland - HIC & University & $\begin{array}{l}\text { Young adults, } \\
N=553\end{array}$ & Not stated & $23.42(3.27)$ \\
\hline Martins et al. [2011] & Portuguese & PT & Cross-sectional & Portugal- HIC & $\begin{array}{l}\text { Clinical \& } \\
\text { Online }\end{array}$ & & Convenience & $32.01(4.65)$ \\
\hline
\end{tabular}


Table 5 Study descriptions (Continued)

\begin{tabular}{|c|c|c|c|c|c|c|c|c|}
\hline $\begin{array}{l}\text { Authors } \\
\text { [Year of publication] }\end{array}$ & Language (s) & Study aim(s) & Design & $\begin{array}{l}\text { Country - } \\
\text { [income bracket] }\end{array}$ & Setting & Participants & Sampling & $\begin{array}{l}\text { Age in years- } \\
\text { Mean [SD] }\end{array}$ \\
\hline & & & & & & $\begin{array}{l}\text { Adults attempting } \\
\text { to get pregnant, } \\
N=312\end{array}$ & & \\
\hline Martins et al. [2012] & Portuguese & $\mathrm{T} \& \mathrm{PT}$ & Longitudinal & Portugal- HIC & $\begin{array}{l}\text { Clinical \& } \\
\text { Online }\end{array}$ & $\begin{array}{l}\text { Adults attempting } \\
\text { to get pregnant, } \\
N=589\end{array}$ & Convenience & $33.8(5.2)$ \\
\hline Martins et al. [2014] & Portuguese & PT & Cross-sectional & Portugal- HIC & $\begin{array}{l}\text { Clinical \& } \\
\text { Online }\end{array}$ & $\begin{array}{l}\text { Adults attempting } \\
\text { to get pregnant, } \\
N=426\end{array}$ & Convenience & $\begin{array}{l}\text { 1.Men- 34.3(6.2) } \\
\text { 2. Women-32.3 } \\
\text { (4.9) }\end{array}$ \\
\hline Guan et al. [2015] & $\begin{array}{l}\text { Simplified } \\
\text { Chinese }\end{array}$ & $T \& P T$ & Longitudinal & China - HIC & University & $\begin{array}{l}\text { University } \\
\text { students, } N=202\end{array}$ & Convenience & $21.9(2.0)$ \\
\hline Cobb \& Xie [2015] & Spanish & PT & Cross-sectional & USA-HIC & Community & $\begin{array}{l}\text { Hispanic } \\
\text { immigrants, } \\
N=122\end{array}$ & Not stated & $33.7(8.2)$ \\
\hline Guillén et al. [2015] & Spanish & PT & Cross-sectional & Spain - HIC & Community & $\begin{array}{l}\text { Female intimate } \\
\text { partner violence } \\
\text { victims, } N=136\end{array}$ & Convenience & $\begin{array}{l}31.67 \\
\text { (SD not stated) }\end{array}$ \\
\hline Ramos et al. [2016] & Spanish & $T \& P T$ & Cross-sectional & Spain - HIC & Community & Retirees, $N=991$ & Convenience & $62.7(5.89)$ \\
\hline Rey et al. [2016] & Spanish & PT & Cross-sectional & Spain - HIC & Community & Adults, $N=613$ & Not stated & $34.36(11.18)$ \\
\hline Trujols et al. [2014] & Spanish & PT & Cross-sectional & Spain - HIC & Clinical & $\begin{array}{l}\text { Patients with } \\
\text { depression, } \\
N=173\end{array}$ & Consecutive & $50.2(14.9)$ \\
\hline Ekbäck et al. [2013] & Swedish & $T \& P T$ & Cross-sectional & Sweden -HIC & Clinical & $\begin{array}{l}\text { 1. Patients with } \\
\text { Hirsutism, } n=127 \\
\text { 2. Nursing } \\
\text { students, } n=154\end{array}$ & Not stated & $\begin{array}{l}\text { 1. Patients with, } \\
\text { Hirsutism, } 32.0 \\
\text { (10.3) } \\
\text { 2. Nursing } \\
\text { students, } 27.3 \\
\text { (7.8) }\end{array}$ \\
\hline Ekbäck et al. [2014] & Swedish & PT & $\begin{array}{l}\text { Comparative, } \\
\text { cross-sectional }\end{array}$ & Sweden -HIC & Clinical & $\begin{array}{l}\text { 1.Patients with } \\
\text { Hirsutism, } n=127 \\
\text { 2. Normative } \\
\text { sample, } n=1115\end{array}$ & Not stated & $\begin{array}{l}\text { 1. Patients with } \\
\text { Hirsutism } 32.0 \\
\text { (10.2) } \\
\text { 2. Normative } \\
\text { sample, } \\
32.7(7.9)\end{array}$ \\
\hline Guan et al. [2013] & Tamil & $T \& P T$ & Cross-sectional & Malaysia-UMIC & University & $\begin{array}{l}\text { University } \\
\text { students, } N=94\end{array}$ & Not stated & $38.3(17.9)$ \\
\hline Ross et al. [2011] & Thai & PT & $\begin{array}{l}\text { Cross-sectional, } \\
\text { correlational }\end{array}$ & Thailand-UMIC & Clinical & $\begin{array}{l}\text { Postpartum, } \\
\text { HIV-positive } \\
\text { women, } N=85\end{array}$ & Convenience & $26.8(5.64)$ \\
\hline Wongpakaran [2011] & Thai & $T \& P T$ & Cross-sectional & Thailand-UMIC & University & $\begin{array}{l}\text { 1. Medical } \\
\text { students, } n=310 \\
\text { 2.Patients with } \\
\text { major depressive } \\
\text { disorder, } n=152 \\
{[N=462]}\end{array}$ & Convenience & $\begin{array}{l}\text { 1. Medical } \\
\text { students, } \\
n=19.16 \text { (1.02) } \\
\text { 2. Patients with } \\
\text { major } \\
\text { depressive } \\
\text { disorder, } 41.23 \\
\text { (12.30) }\end{array}$ \\
\hline Wongpakaran [2012] & Thai & $\mathrm{T} \& \mathrm{PT}$ & Cross sectional & Thailand-UMIC & University & $\begin{array}{l}\text { Medical students, } \\
N=486\end{array}$ & Not stated & $19.01(0.90)$ \\
\hline Eker \& Arkar [1995] & Turkish & $T \& P T$ & Cross-sectional & Turkey - UMIC & $\begin{array}{l}\text { University \& } \\
\text { Clinical }\end{array}$ & $\begin{array}{l}\text { 1. University } \\
\text { students, } n=146 \\
\text { 2. Patients with } \\
\text { renal problems, } \\
n=50\end{array}$ & Not stated & $\begin{array}{l}\text { 1. University } \\
\text { students, } 20.34 \\
\text { (1.55) } \\
\text { 2. Patients with } \\
\text { renal } \\
\text { problems,37.18 } \\
\text { (12.8) }\end{array}$ \\
\hline Ersoy \& Varan [2007] & Turkish & PT & Cross-sectional & Turkey - UMIC & Clinical & $\begin{array}{l}\text { Patients with } \\
\text { psychiatric } \\
\text { disorders, } N=203\end{array}$ & Convenience & $33.79(11.77)$ \\
\hline $\begin{array}{l}\text { Eker, Arkar \& Yaldiz } \\
\text { [2000] }\end{array}$ & Turkish & PT & Cross-sectional & Turkey - UMIC & Clinical & $\begin{array}{l}\text { 1. Psychiatry } \\
\text { patients, } n=502 \text {. } \\
\text { Surgery patients, } \\
n=503 \text {. } \\
\text { Normative } \\
\text { sample, } n=50\end{array}$ & Convenience & $\begin{array}{l}\text { 1. Psychiatry } \\
\text { patients, 36(13) } \\
\text { 2. Surgery } \\
\text { patients, 36(13) } \\
\text { 3. Normative } \\
\text { sample, 35(11) }\end{array}$ \\
\hline
\end{tabular}


Table 5 Study descriptions (Continued)

\begin{tabular}{|c|c|c|c|c|c|c|c|c|}
\hline $\begin{array}{l}\text { Authors } \\
\text { [Year of publication] }\end{array}$ & Language (s) & Study aim(s) & Design & $\begin{array}{l}\text { Country - } \\
\text { [income bracket] }\end{array}$ & Setting & Participants & Sampling & $\begin{array}{l}\text { Age in years- } \\
\text { Mean [SD] }\end{array}$ \\
\hline Kuscu et al. [2009] & Turkish & PT & Cross-sectional & Turkey - UMIC & Clinical & $\begin{array}{l}\text { Caregivers of } \\
\text { adult cancer } \\
\text { patients, } N=51\end{array}$ & Convenience & Not stated \\
\hline Duru [2007] & Turkish & PT & Cross-sectional & Turkey - UMIC & University & Students, $N=340$ & Not stated & $18.83(1.35)$ \\
\hline Basol [2008] & Turkish & PT & Cross-sectional & Turkey - UMIC & Schools & $\begin{array}{l}\text { Administrators, } \\
N=433\end{array}$ & Not stated & Not stated \\
\hline Akhtar et al. [2010] & Urdu & PT & Longitudinal & Pakistan- LMIC & Community & $\begin{array}{l}\text { Antenatal women, } \\
N=325\end{array}$ & Not stated & $27(5)$ \\
\hline Saleem et al. [2013] & Urdu & PT & Cross sectional & Pakistan- LMIC & Clinical & $\begin{array}{l}\text { Drug addicts, } \\
N=70\end{array}$ & Not stated & $32.21(8.30)$ \\
\hline Khan et al. [2015] & Urdu & PT & Cross sectional & Pakistan- LMIC & Community & $\begin{array}{l}\text { Pregnant women, } \\
N=349\end{array}$ & Cluster & $\begin{array}{l}<19-30+ \\
\text { [Range] }\end{array}$ \\
\hline Qadir et al. [2013] & Urdu & PT & Cross sectional & Pakistan- LMIC & Community & $\begin{array}{l}\text { Married women, } \\
N=277\end{array}$ & Convenience & 36.7 (9.96) \\
\hline Naveed \& Naz [2015] & Urdu & PT & Cross sectional & Pakistan- LMIC & Clinical & $\begin{array}{l}\text { Women with } \\
\text { postpartum } \\
\text { depression, } \\
N=100\end{array}$ & Not stated & $27.31(5.20)$ \\
\hline
\end{tabular}

T- translation: PT psychometric testing, LIC lower income country, LMIC lower-middle income country, UMIC upper middle-income country, HIC high income country $<$ World Bank Classification system $>$

of the translators were not clearly described; it was not clear if the translations were done independently and the tool was not pretested. The evidence for criterion validity, construct validity and reliability was indeterminate. No information was provided on the psychometric robustness of comparison outcome measures, the time for the re-test was inappropriate and no specific hypotheses were formulated.

\section{Creole}

Only one study was available [6]. The tool was poorly translated as; there were no multiple translations, the tool was not pre-tested and factorial analysis was not done. The evidence for IC was indeterminate as the subscales unique ICs were not computed and handling of missing responses was not documented. There was indeterminate evidence for reliability as the conditions for the administrations were not clearly stated and the evidence for no systematic change in the outcomes was not provided.

\section{French}

Only one study was available [9]. The cross-cultural translation and adaptation process was poor. The tool was not pre-tested and only solitary forwardbackward translations were performed. There was limited evidence for structural validity, IC, reliability and construct validity. The methodologies applied were of fair quality, the handling of missing response was not reported, the conditions for the test-retest were not clearly outlined and no specific hypotheses were formulated respectively.

\section{Hausa}

Three studies were available [7, 14, 85]. The methodology for the cross-cultural validation was good. A solitary backward translation was done and reconciliation process was poorly described. There was strong evidence for structural validity and IC. There was limited evidence for test-retest reliability and construct validity. It was not clear if administrations were independent, if patients were stable in between administrations and the MSPSS was re-administered after a week against the recommended 2 weeks [57, 86]. Further, the handling of missing responses was not reported and no specific hypotheses were formulated.

\section{Korean}

One study was available [87]. The methodology for the cross-cultural validation was poor. There was a scanty description of the expertise of the translators and whether the translations were done independently. It was not clear if the tool was pretested in the target population and solitary forward and backward translations were performed. There was poor evidence for structural validity as only EFA was performed. There was strong evidence for IC as the methodology was of excellent quality. There was limited evidence for construct validity, no specific hypotheses were formulated.

\section{Luganda}

One study was available [13]. The methodology for the cross-cultural validation was fair. A solitary forward translation was performed; the characteristics of the pretest sample were not clearly described and details of the reconciliation of the original and forward translation 


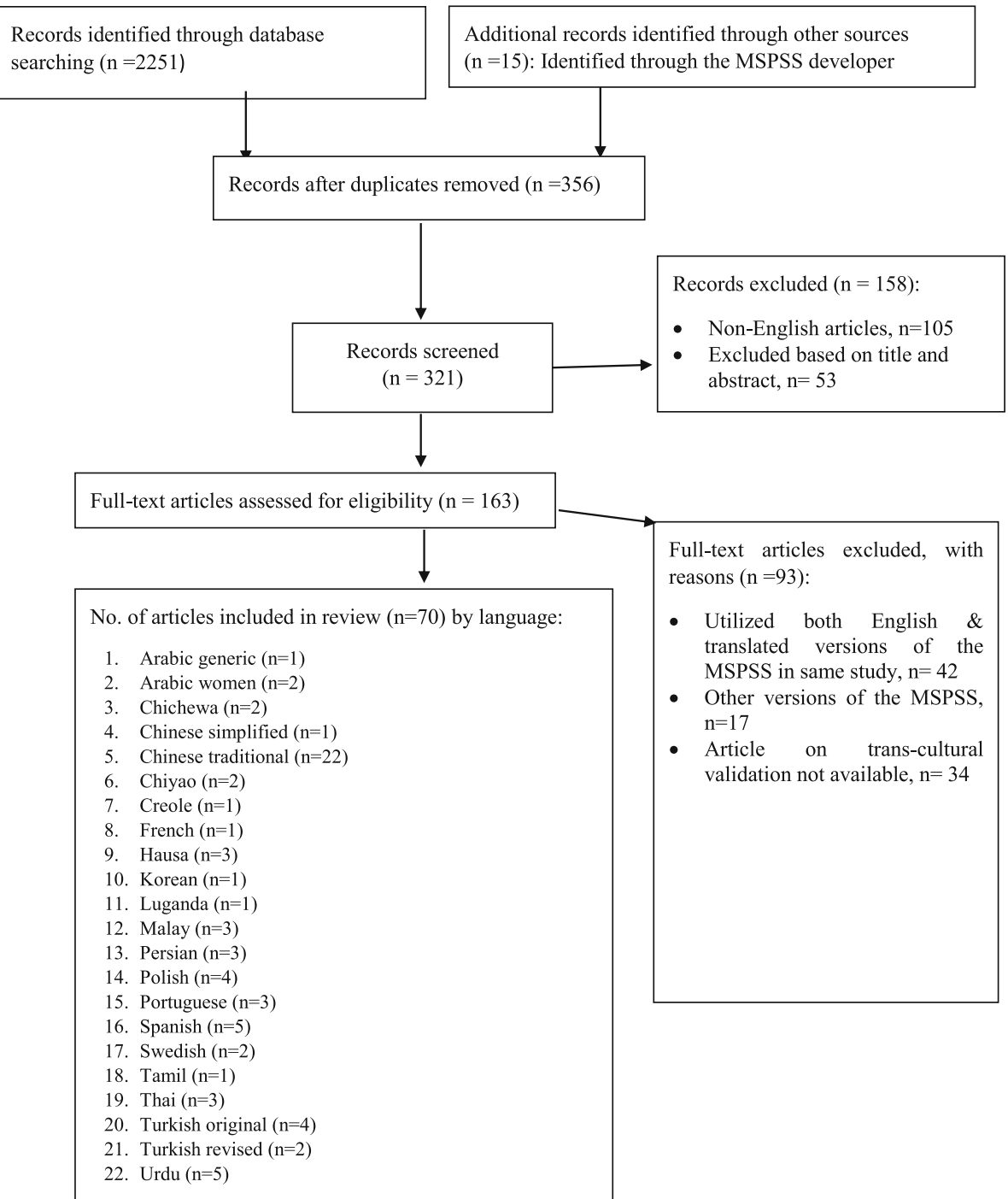

Fig. 1 Flowchart of article search and selection process: We identified 2251 articles, of which 356 were duplicates. After applying the selection criterion, 70 articles were analysed for the present review

were scanty. There was unknown evidence for structural validity as only EFA was performed. Further, an inappropriate rotation method (orthogonal rotation) was applied for EFA. There was limited evidence of the IC, the handling of missing responses was not documented.

\section{Malay}

Three studies were available $[11,88,89]$. The methodology for the cross-cultural validation was poor. There was a scanty description of; the expertise of the translators, whether the translations were done independently, the reconciliation process, and the tool was not pretested in the target population. There was poor evidence for structural validity as only EFA was performed. There was indeterminate evidence for IC, the handling of missing responses was not documented. There was unknown evidence for construct validity; no specific hypotheses were formulated with poor/no description of the psychometrics of the comparator instruments. There was no report of test-retest reliability; the stability of the respondents was not clearly outlined; the tool was readministered after a week and there was a disparity in administration conditions as the items were reshuffled for the retest. There was unknown evidence for criterion validity, the psychometrics for the purported "gold standard" outcome measure was not provided.

\section{Persian}

Three studies were available $[18,90,91]$. The methodology for the cross-cultural validation was poor. The expertise of translators, handling of missing responses and 
Table 6 Adaptations to the MSPSS and outcome measures per study

\begin{tabular}{|c|c|c|c|c|c|c|c|}
\hline \multirow[t]{2}{*}{ Language (s) } & \multirow{2}{*}{$\begin{array}{l}\text { esponse } \\
\text { options }\end{array}$} & \multirow[t]{2}{*}{ Modification } & \multirow{2}{*}{$\begin{array}{l}\text { Mode of } \\
\text { administration }\end{array}$} & \multirow{2}{*}{$\begin{array}{l}\text { Statistical } \\
\text { analyses }\end{array}$} & \multicolumn{3}{|c|}{ Secondary outcome measures } \\
\hline & & & & & Measure 1 & Measure 2 & Measure 3 \\
\hline Arabic & 3 & $\begin{array}{l}\text { Special person } \\
\text { changed to } \\
\text { Husband }\end{array}$ & $\begin{array}{l}\text { Interviewer } \\
\text { administered }\end{array}$ & CFA & $\begin{array}{l}\text { Seeking Social } \\
\text { Support, Problem } \\
\text { Solving, Blaming } \\
\text { Self and Avoidance } \\
\text { Scales - Revised } \\
\text { Ways of Coping } \\
\text { Checklist (RWCCL) }\end{array}$ & $\begin{array}{l}\text { Emotional distress- Profile } \\
\text { of Mood States (POMS) }\end{array}$ & $\begin{array}{l}\text { Daily Hassles } \\
\text { Scale (DHS) }\end{array}$ \\
\hline Arabic & 7 & None & Not stated & EFA & $\begin{array}{l}\text { Emotion } \\
\text { Regulation } \\
\text { Questionnaire } \\
\text { (Arabic-ERQ) }\end{array}$ & $\begin{array}{l}\text { Satisfaction with Life } \\
\text { Scale (Arabic-SWLS) }\end{array}$ & \\
\hline $\begin{array}{l}\text { Chichewa } \\
(n=269) \& \\
\text { Chiyao }(n=314)\end{array}$ & 5 & $\begin{array}{l}\text { Questions changed } \\
\text { to second person } \\
\text { pronouns }\end{array}$ & $\begin{array}{l}\text { self-administered \& } \\
\text { interviewer } \\
\text { administered }\end{array}$ & $\begin{array}{l}\text { 1. EFA } \\
\text { 2. CFA }\end{array}$ & $\begin{array}{l}\text { Depression - Self } \\
\text { Reporting } \\
\text { Questionnaire (SRQ) }\end{array}$ & $\begin{array}{l}\text { Depression - } \\
\text { Edinburgh Postnatal } \\
\text { Depression Scale (EPDS) }\end{array}$ & $\begin{array}{l}\text { Depression - } \\
\text { Structured } \\
\text { Clinical } \\
\text { Interview for } \\
\text { DSM-IV (SCID) }\end{array}$ \\
\hline Creole & 7 & none & Self-administered & $\begin{array}{l}\text { Parametric \& } \\
\text { non-parametric } \\
\text { tests }\end{array}$ & $\begin{array}{l}\text { Perceived } \\
\text { Adequacy of } \\
\text { Resource Scale } \\
\text { (PARS) }\end{array}$ & & \\
\hline French & 7 & None & Not stated & CFA & $\begin{array}{l}\text { Depression - } \\
\text { Edinburgh } \\
\text { Postnatal } \\
\text { Depression Scale } \\
\text { (EPDS) }\end{array}$ & & \\
\hline Hausa & 7 & $\begin{array}{l}\text { Terms changed to } \\
\text { culturally acceptable } \\
\text { equivalents }\end{array}$ & $\begin{array}{l}\text { Interviewer } \\
\text { administered }\end{array}$ & Qualitative & & & \\
\hline Hausa & 7 & None & Not stated & CFA & & & \\
\hline Hausa & 7 & None & Not stated & Regression & $\begin{array}{l}\text { Disability- modified } \\
\text { Rankin Scale }\end{array}$ & & \\
\hline Simplified Chinese & 4 & Not clearly stated & Self-administered & EFA & $\begin{array}{l}\text { Psychological } \\
\text { symptomatology - } \\
\text { GHQ } 30\end{array}$ & $\begin{array}{l}\text { Social networks- Lubben } \\
\text { Social Network Scale }\end{array}$ & \\
\hline Simplified Chinese & 5 & None & Self-administered & CFA & & & \\
\hline Korean & 7 & $\begin{array}{l}\text { Significant other } \\
\text { replaced by } \\
\text { "spouse/partner" }\end{array}$ & Not stated & EFA & $\begin{array}{l}\text { Perceived social } \\
\text { support- Personal } \\
\text { Resource } \\
\text { Questionnaire -2 } \\
\text { (PRQ) }\end{array}$ & $\begin{array}{l}\text { Self-efficacy- Self -efficacy } \\
\text { for Diabetes Scale }\end{array}$ & $\begin{array}{l}\text { Depression- } \\
\text { Center for } \\
\text { Epidemiological } \\
\text { Studies } \\
\text { Depression } \\
\text { Scale (CEDS) }\end{array}$ \\
\hline Luganda & 5 & use of facial cues & $\begin{array}{l}\text { Interviewer } \\
\text { administered }\end{array}$ & EFA & & & \\
\hline Malay & 7 & Not clearly stated & Self-administered & EFA & $\begin{array}{l}\text { Social support - } \\
\text { Medical Outcome } \\
\text { Survey (MOS) }\end{array}$ & Depression- BDI & $\begin{array}{l}\text { Mental health- } \\
\text { General Health } \\
\text { Questionnaire }\end{array}$ \\
\hline Persian & 7 & Not clearly stated & Not stated & EFA & & & \\
\hline Polish & 7 & None & Self-administered & $\begin{array}{l}\text { 1. EFA } \\
\text { 2. CFA }\end{array}$ & $\begin{array}{l}\text { Loneliness- The } \\
\text { Social and } \\
\text { Emotional } \\
\text { Loneliness Scale for } \\
\text { Adults-Short Form } \\
\text { (SELSA-S) }\end{array}$ & $\begin{array}{l}\text { The State-Trait Anxiety } \\
\text { Inventory (STAI) }\end{array}$ & $\begin{array}{l}\text { Satisfaction with } \\
\text { Life Scale } \\
\text { (SWLS) }\end{array}$ \\
\hline Portuguese & 6 & None & Self-administered & CFA & $\begin{array}{l}\text { Depression - Beck } \\
\text { Depression } \\
\text { Inventory }\end{array}$ & $\begin{array}{l}\text { Fertility- Fertility } \\
\text { Problem Inventory }\end{array}$ & \\
\hline Simplified Chinese & 7 & None & Self-administered & EFA & & Depression - BDI & \\
\hline
\end{tabular}


Table 6 Adaptations to the MSPSS and outcome measures per study (Continued)

\begin{tabular}{|c|c|c|c|c|c|c|c|}
\hline \multirow[t]{2}{*}{ 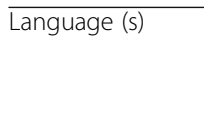 } & \multirow{2}{*}{$\begin{array}{l}\text { esponse } \\
\text { options }\end{array}$} & \multirow[t]{2}{*}{ Modification } & \multirow{2}{*}{$\begin{array}{l}\text { Mode of } \\
\text { administration }\end{array}$} & \multirow{2}{*}{$\begin{array}{l}\text { Statistical } \\
\text { analyses }\end{array}$} & \multicolumn{3}{|c|}{ Secondary outcome measures } \\
\hline & & & & & Measure 1 & Measure 2 & Measure 3 \\
\hline & & & & & $\begin{array}{l}\text { Social support - } \\
\text { Medical Outcome } \\
\text { Survey (MOS) }\end{array}$ & & $\begin{array}{l}\text { Mental health- } \\
\text { General Health } \\
\text { Questionnaire }\end{array}$ \\
\hline Spanish & 7 & None & Self-administered & EFA & None & None & none \\
\hline Swedish & 7 & None & Self-administered & EFA & & & \\
\hline Tamil & 7 & None & Self-administered & EFA & $\begin{array}{l}\text { Social support - } \\
\text { Medical Outcome } \\
\text { Survey (MOS) }\end{array}$ & $\begin{array}{l}\text { Depression - Beck } \\
\text { Depression Inventory }\end{array}$ & $\begin{array}{l}\text { Mental health- } \\
\text { General Health } \\
\text { Questionnaire }\end{array}$ \\
\hline Thai & 7 & None & Self-administered & $\begin{array}{l}\text { 1.EFA } \\
\text { 2.CFA }\end{array}$ & $\begin{array}{l}\text { Anxiety- The Sate } \\
\text { Trait Anxiety } \\
\text { Inventory (STAI) }\end{array}$ & $\begin{array}{l}\text { Self-esteem-The } \\
\text { Roseburg Self-Esteem } \\
\text { Scale (RSES) }\end{array}$ & $\begin{array}{l}\text { Depression- } \\
\text { Thai Depression } \\
\text { Scale (TDS) }\end{array}$ \\
\hline Traditional Chinese & 7 & Not clearly stated & $\begin{array}{l}\text { Interviewer } \\
\text { administered }\end{array}$ & $\begin{array}{l}\text { 1. EFA } \\
\text { 2. CFA }\end{array}$ & & & \\
\hline Turkish & 7 & None & Not stated & EFA & $\begin{array}{l}\text { Depression - Beck } \\
\text { Depression } \\
\text { Inventory }\end{array}$ & $\begin{array}{l}\text { The State-Trait Anxiety } \\
\text { Inventory (STAI) }\end{array}$ & \\
\hline Urdu & 7 & None & $\begin{array}{l}\text { Interviewer } \\
\text { administered }\end{array}$ & EFA & $\begin{array}{l}\text { Mental distress- } \\
\text { Self Report } \\
\text { Questionnaire } \\
\text { (SRQ-20) }\end{array}$ & Depression - EPDS & \\
\hline
\end{tabular}

reconciliation process was poorly described. Further, solitary forward and backward translations were performed and the tool was not pre-tested. There was poor evidence for structural validity as only EFA was performed. There was limited evidence for IC and reliability as the methodologies were of fair quality. Only 71 participants were recruited for test re-test reliability and the conditions and stability for the re-test sample were not clearly stared. There was unknown evidence for construct validity as no specific hypotheses were formulated and no the psychometrics of the comparator instruments were not provided.

\section{Polish}

Four studies were available [92-95]. The methodology for the cross-cultural validation was good. The reconciliation of the translations was poorly described and the tool was not reviewed by a committee. There was strong evidence for IC and structural validity. There was moderate, negative evidence for construct validity as no specific hypotheses were formulated.

\section{Portuguese}

Three studies were available [31, 96, 97]. The methodology for the cross-cultural validation was fair. The expertise of the translators was not stated, if was not clear if translations were done independently, only solitary forward and backward translations were done and the tool was not reviewed by a committee. There was excellent evidence for both structural validity and IC. There was unknown evidence for test-retest reliability; a sub-optimal sample $(n=52)$ was utilized, the stability of the participants and the conditions for the re-test were not stated. There was limited evidence for construct validity as no specific hypotheses were formulated.

\section{Spanish}

Five studies were available [34, 98-101]. The methodology for the cross-cultural validation was poor. The expertise of the translators was not stated; only solitary forward and backward translations were done and the tool was not pre-tested. There was conflicting evidence for structural validity as the cited studies were of both poor and fair quality. For instance, for one of the studies, EFA contrary to the CFA reported was done and authors performed varimax (orthogonal) rotation [101]. There was limited evidence for IC as the handling of missing responses was not recorded. There was moderate evidence for construct validity as no specific hypotheses were formulated.

\section{Swedish}

Two studies were available [3, 102]. The methodology for the cross-cultural validation was poor. A solitary backward translation was performed; the handling of missing responses was not reported and the credentials of the translators were not clearly described. Evidence for structural validity was poor, only EFA was performed and an inappropriate rotation method (orthogonal vari$\max$ ) was utilized. There was moderate evidence for IC, the handling of missing responses was not described. 
Evidence for reliability was limited as a sub-optimal sample size $(n=44)$ was used for the retest and the conditions of the re-test administration were not clearly described. There was limited evidence for construct validity as no specific hypotheses were formulated.

\section{Tamil}

Only one study was available [103]. The methodology for the cross-cultural validation poor. There was scanty description of: the expertise of the translators was, if forward translations were done independently, the reconciliation process, whether the tool was not pre-tested in the target population and the profile of the pre-test sample. There was limited evidence for IC, a sub-optimal sample size $(N=94)$ was recruited and handling of missing responses was not reported. There was unknown evidence for structural validity, construct validity and criterion validity. Only EFA was performed, no specific hypotheses were formulated and the psychometrics of the purported "gold standard" were not provided.

\section{Thai}

Three studies were available $[28,43,44]$. The methodology for the cross-cultural validation was poor. The forward translators did not work independently; only solitary forward and backward translations were performed and scanty details were provided for the reconciliation process and the pre-test sample profile. There was moderate evidence for structural validity and IC. Both EFA and CFA were performed, however, the percentage of missing responses was not stated. There was unknown evidence for construct validity as the no specific hypothesis were formulated. There was limited evidence for test-retest reliability, a suboptimal sample $(N=72)$ was utilized, the conditions and stability of patients for the re-test were not clearly outlined.

\section{Turkish}

Two versions of the Turkish translations were available i.e. the original Turkish version $[39,41]$ and the revised Turkish version $[29,104]$.

\section{Original Turkish version}

Four studies were available [39-42]. The methodology for the cross-cultural validation was poor. A solitary forward translation was performed; it is not clear if the forward translators worked independently and the tool was not pre-tested. There was poor evidence for structural validity as CFA was not performed. There was moderate evidence for IC, the handling of missing responses was not described. There was unknown evidence for construct validity as no specific hypotheses were formulated and one of the studies was of poor methodological quality [41].

\section{Revised Turkish version}

Two studies were available $[29,104]$. There was moderate evidence for structural validity as the handling of missing values was not described. Evidence was; conflicting for IC and unknown for reliability and construct validity. A sub-optimal sample was utilized; the test conditions and stability of the participants was neither described and no specific hypotheses were formulated.

\section{Urdu}

Five studies were available [45-49]. The methodology for the cross-cultural validation was poor. The following were not stated; the expertise of the translators, if translations were done independently, the number of forward and backward translations. Further, the tool was not reviewed by a committee and was not pretested in the target population. There was limited evidence for structural validity [47, 49]. One of the studies was of poor quality, only EFA was performed [49]. For the second study, although CFA was performed, the handling of missing responses was not documented [47]. There was indeterminate evidence for IC as the methodology was of poor quality. There was moderate evidence for construct validity, no specific hypotheses were formulated.

\section{Discussion \\ Settings}

The MSPSS has been translated across a range of settings and populations.

\section{Translation quality}

Trans-cultural adaptation, translation and validation aim to succinctly capture the meaning of a latent construct in another population. As such, a rigorous translation process is essential $[25,52]$. None of the studies included in this review were translated using robust methodologies, with 16 of the 22 studies being of poor methodological quality in accordance with the COSMIN criteria [56]. The lack of quality of the translations affects the generalizability and comparability of the study findings. For example, if the MSPSS is applied in a large multinational trial, there is risk of misleading results if one of the translations was poorly conducted. The findings could have negative implications on policy formulation, over-/under estimation of an intervention effect size amongst other.

The lack of detailed descriptions of both language and construct expertise of the translators, whether the translations were done independently and reconciliation of the translations compromised the methodological rigor of most of the retrieved studies. Furthermore, the 
absence of a panel of experts review process for content and face validation, as was the case in 13 of the 21 included studies, could have jeopardised the ability to produce a culturally acceptable translation [25, 52]. Ideally, the panel should consist of experts with diverse professional backgrounds to ensure the attainment of semantic, idiomatic, and conceptual equivalence [25]. For instance, given the differences in cultures, the interpretation of the term "special person" can vary from setting to setting. It is argued that respondents from collectivistic cultures may not distinguish between family and a significant other as sources of SS [47, 49]. For example, in Turkey, when the term "special person" was changed to "husband" following a panel of experts' review, the resultant/revised translation yielded a three-factor structure as opposed to the earlier two-factor structure [29, 104]. This illustrates that a more rigorous reconciliation and adaptation can yield a more reliable factor structure. Lastly, pretesting/cognitive debriefing of the translated and adapted tool is essential before the tool can be applied to a larger population $[25,52,56]$. This should be done in the target population as translation is an integrated and iterative process and requires input from "experts" and the "target users" of the PROM [52]. Unfortunately, only five of the 23 translations described this process in detail, including description of sample selection, hence this could also be a source of methodological limitation for the retrieved studies.

\section{Structural validity}

Structural/factorial validity is defined as the extent to which scores on an outcome measure adequately reflect the dimensions/structure of the construct to be measured [41]. Factorial validity can be envisaged as the 'backbone' for the statistical evidence of the validity or lack thereof of a translated tool. Ideally for translated outcome measures, both EFA and CFA should be performed to test factorial validity $[3,31]$. EFA is a technique used to explore/discover the number of factors a tool possesses [105-107]. The original MSPSS has a three-factor structure, it is essential to test if this is the same for the translated versions as SS is a multidimensional, subjective construct which is dependent on sociocultural contextual factors $[1,3,10-12,17,18,108]$. To this end, it is acceptable to obtain a one- or two-factor structured translation if the translation method is adequately robust. However, EFA alone is inadequate, as was the case in most of the retrieved translations; therefore, CFA ought to also have been performed [57, 86]. CFA is an advanced structural equation modelling statistical technique which combines the concepts of EFA, correlation and multiple regression $[109,110]$. It provides evidence as to whether the translated versions replicated the original three-factor structure as postulated by the developers of the MSPSS. Unfortunately, a minority of the studies [9/23] performed CFA with only four studies [4/23] performing both EFA and CFA which is a major shortcoming for the level of evidence for structural validity. Further, in some instances, some authors/studies refer to EFA as CFA [28, $43,44,103]$ and this again yields inaccurate conclusions. In instances were only EFA was performed, some authors utilized an inappropriate rotational method i.e. orthogonal instead of oblique rotation $[3,13,102]$. Orthogonal rotation is used when the factors are hypothesized to be unrelated [105, 107, 111], which is not the case for the MSPSS as the domains are stipulated to be correlated $[15,21,22]$. For studies which performed CFA, only three adequately described the goodness of fitness (GOF) indices. These are important as they provide concrete evidence to the degree to which the data/translation fits into the original factor model $[109,110,112]$. Furthermore, given that the MSPSS can yield one-, two- or three- factors, all the three models should be tested using CFA before a decision on the degree of fit can be made. None of the studies which performed both EFA and CFA included this analysis, hence this could be envisaged as a potential source of reporting bias. Replication of the original factorial structure is not necessarily a benchmark for an accurate translation process [25]. For instance, authors may be tempted not to report the results of a two-factor model if the degree of fit is much better than for a three-factor model. Provision of multiple GOF indices for all the three models should be a "standard" reporting practise as it provides the potential readership with all the essential information for them to critique the methodological quality and subsequent conclusions in keeping with the evidence supplied [110].

\section{Reliability}

Most of the translated tools displayed adequate evidence for IC as most attained a Cronbach's alpha of at least 0 . 70. However, given the limitations in the structural validity testing and lack of rigour in the translation process, the results for IC may need to be interpreted with caution. This is because a tool can be reliable, yet not valid $[25,113]$. To illustrate this, if only EFA is performed, the factorial validity will be poor, however, the tool can still yield a high alpha statistic. In that instance the reliability findings can be deemed as "misleading" [114]. The validity of the alpha scores is also dependent on the homogeneity/unidimensionality of a test and this can be established through factor analysis [56]. Therefore, if CFA (the preferred unidimensionality test for translated tools) is not performed, the IC for that test will/may not be valid $[56,114,115]$. Additionally, the longitudinal validity (test-retest) also gives further evidence of the stability of an outcome measure over time [56]. Only four studies reported the stability of the translated versions which is another potential limitation. Given the potential 
limitation in relying solely on the IC as an indicator of reliability, other indices such as the alternative forms, split-half and test-retest reliability are recommended for concrete evidence of reliability of outcome measures $[113,115]$. More so, it is argued that despite its wide usage and popularity, the Cronbach alpha is least desirable index for reliability estimation [115].

\section{Construct validity}

Construct validity refers to the extent to which scores on an instrument relate to other measures in a manner that is consistent with theoretically derived hypotheses concerning the concepts that are being measured [54]. Depression, anxiety, self-esteem and general mental well-being were the most commonly reported outcomes against which SS scores were compared. To prevent report bias, the developers of the COSMIN checklist recommend that authors should formulate specific hypothesis before data collection [54, 57, 86]. None of the studies specified the expected magnitude of correlations with only three studies formulating specific hypotheses. Further, there is need for authors to describe in detail the comparator instruments as well as demonstrating their reliability and validity in the study population as failure to do so affects the both internal and external validity [86]. For example, some of the translations [eight out of twenty-three] did not report the psychometrics of the comparator instruments. In other instances, the authors refer to the psychometrics of the comparator from another population which again is questionable [45-49]. Failure to demonstrate the psychometric robustness of the comparator instruments would thus affect the construct validity of the translated versions of the MSPSS.

\section{Criterion validity}

Criterion validity is defined as the extent to which scores on an outcome measure perform against an established gold standard [58]. Given that SS is a latent variable [3, 11], it is difficult to establish a gold standard against which the MSPSS can be assessed against. Nevertheless, for the three studies which evaluated criterion validity, the psychometrics of the purported gold standard were either questionable or were not well described. Therefore, there was poor evidence for criterion validity.

\section{Limitations}

The use of the COSMIN checklist for the evaluation of the methodological quality may have been a potential limitation. This is because the checklist came into effect in 2011 and some of the translations had been performed prior to its' publication. The stringent nature of the checklist has also been reported in almost similar systematic reviews $[24,116]$. For example, in the assessment of IC and factorial validity, if the handling of missing responses is not reported, the domain(s) are rated as fair quality despite the rest of the ratings being of excellent quality. Inconsistencies within the COSMIN checklist may also be viewed as a potential source of limitation. For example, in evaluating the structural validity of translated tools, if CFA is not performed, item 6 for the structural validity/Box E is rated as good and the same is rated as poor for item 14 under Box G/cross-cultural validity. As the COSMIN guidelines are currently under review, it is hoped the revised guidelines will further harmonize the terminology utilized in the methodological and further increase the checklist validity in rating methodological quality of the translation and adaptation of PROMs. Additionally, we could not evaluate fifteen language versions of the MSPSS which were published in other than English language and this could have introduced language bias for the present review.

\section{Conclusions}

We identified 22 translated versions of the MSPSS. The psychometric properties which were most often reported included internal consistency, test-retest reliability, structural validity and construct validity. Many of the tools did not follow a rigorous translation process and there was poor evidence for structural validity. The advent of EBP and increased usage of PROMs requires quality translations to ensure reliable and valid outcome measures. The retrieved MSPSS translations therefore need to be utilized with precautions. There is also need to assess other psychometric properties such as responsiveness, measurement error and establishment of cutoff values to increase the clinical utility and psychometric robustness of the translated versions of the MSPSS. We also recommend the development of a standardized protocol for the translation and adaptation of the MSPSS. Future translation studies should utilize the backward-forward translation method with special emphasis on the use of multiple translators, reconciliation of translations, panel of expert assessment and both EFA and CFA should be performed for factorial analysis.

\section{Additional files}

Additional file 1: Multidimensional Scale of Perceived Social Support [MSPSS]. (DOC $35 \mathrm{~kb})$

Additional file 2: Populated PRISMA 2009 Checklist. (DOC 63 kb)

\section{Abbreviations}

CFA: Confirmatory factor analysis; COSMIN: COnsensus-based Standards for the selection of health status Measurement Instruments; EBP: Evidence based practise; EFA: Exploratory factor analysis; HIC: High-income country; IC: Internal consistency; LIC: Low income country; LMIC: Lower-middle income country; MIC: Middle income country; MSPSS: Multidimensional Scale 
of Perceived Social Support; PROMs: Patient-reported outcome measures; SS: Social support; UMIC: Upper middle income country

\section{Acknowledgements}

The assistance by Mrs. Morgan from the University of Cape Town library in formulating the search strategy is greatly appreciated.

\section{Disclosure statement}

The views expressed in this publication are those of the author(s) and not necessarily those of AAS, NEPAD Agency, Wellcome Trust, or the UK government.

\section{Funding}

The systematic review is part of the primary authors' PhD work at the University of Cape Town. The work is being funded by The African Mental Health Research Initiative (AMARI). AMARI is a consortium of four African universities whose overall goal is to build excellence in leadership, training and science amongst African scholars in mental, neurological and substance use (MNS) research in Ethiopia, Malawi, South Africa and Zimbabwe. AMARI, at the University of Zimbabwe College of Health Sciences (UZCHS) secured funding from the Wellcome Trust through the Developing Excellence in Leadership and Science (DELTAS) Africa initiative. The DELTAS Africa Initiative is an independent funding scheme of the African Academy of Sciences (AAS)'s Alliance for Accelerating Excellence in Science in Africa (AESA) and supported by the New Partnership for Africa's Development Planning and Coordinating Agency (NEPAD Agency) with funding from the Wellcome Trust [DEL-15-01] and the UK government.

\section{Availability of data and materials}

The data will not be shared since it forms part of ongoing research.

\section{Authors' contributions}

JMD- conceptualized the systematic review, developed the search strategy, ran database searches and drafted the manuscript. JJ - conceptualized the systematic review and edited the manuscript. MC, LC TM - data extraction and editing of the manuscript. $\mathrm{HJ}$ - editing of the manuscript. All authors read and approved the final version of the manuscript.

\section{Ethics approval and consent to participate}

This study was conducted as systematic review, so no ethical approval was sought and there was no need for informed consents.

\section{Competing interests}

The authors declare that they have no competing interests.

\section{Publisher's Note}

Springer Nature remains neutral with regard to jurisdictional claims in published maps and institutional affiliations.

\section{Author details}

'Division of Physiotherapy, School of Health and Rehabilitation Sciences, University of Cape Town, Observatory, Cape Town, South Africa. ${ }^{2}$ Rehabilitation Department, College of Health Sciences, University of Zimbabwe, P.O Box A178, Avondale, Harare, Zimbabwe. ${ }^{3}$ King's College London, Institute of Psychiatry, Psychology, and Neuroscience, London, England. ${ }^{4}$ Harvard Medical School, Boston, MA, USA.

\section{Received: 1 June 2017 Accepted: 24 April 2018}

\section{Published online: 02 May 2018}

\section{References}

1. Stewart RC, Umar E, Tomenson B, Creed F. Validation of the multidimensional scale of perceived social support (MSPSS) and the relationship between social support, intimate partner violence and antenatal depression in Malawi. BMC Psychiatry. 2014;14:180.

2. Raina P, Donnell MO, Schwellnus H, Rosenbaum P, King G, Brehaut J, et al. Caregiving process and caregiver burden: conceptual models to guide research and practice. BMC Pediatr. 2004;4:1-13.

3. Ekbäck M, Benzein $E$, Lindberg M, Årestedt K. The Swedish version of the multidimensional scale of perceived social support (MSPSS) - a psychometric evaluation study in women with hirsutism and nursing students. Health Qual Life Outcomes. 2013:11:168.

4. Wilson A, Yendork JS, Somhlaba NZ. Psychometric properties of multidimensional scale of perceived social support among Ghanaian adolescents. Child Indic Res. 2016;10:101.

5. Aroian K, Templin TN, Ramaswamy V. Adaptation and psychometric evaluation of the multidimensional scale of perceived social support for Arab immigrant women. Health Care Women Int. 2010;31:153-69.

6. Hannan J, Alce M, Astros A. Psychometric properties of the newly translated creole multidimensional scale of perceived social support (MSPSS) and perceived adequacy of resource scale (PARS) and the relationship between perceived social support and resources in Haitian mothers in the USA. BMC Psychol. 2016:4:7.

7. Hamza A, Mohsein NAA, Siew YL. Measuring percieved social support in stroke survivors: linguistic validation of the multidimensional scale of Percieved social support ( MSPSS) in Hausa ( Nigerian ) language. South Afr J Occup Ther. 2012:42.

8. Pedersen SS, Spinder H, Erdman R a M, Denollet J. Poor perceived social support in implantable cardioverter defibrillator (ICD) patients and their partners: cross-validation of the multidimensional scale of perceived social support. Psychosomatics. 2009:50:461-7.

9. Denis A, Callahan S, Bouvard M. Evaluation of the French version of the multidimensional scale of perceived social support during the postpartum period. Matern Child Health J. 2015;19:1245-51.

10. Shvedko A, Whittaker AC, Thompson JL, Greig CA. Physical activity interventions for treatment of social isolation, loneliness or low social support in older adults: A systematic review and meta-analysis of randomised controlled trials. Psychol Sport Exerc [Internet]. Elsevier; 2018;34: 128-37. Available from: https://doi.org/10.1016/j.psychsport.2017.10.003

11. $\mathrm{Ng} \mathrm{CG}$, Amer Siddiq AN, Aida SA, Zainal NZ, Koh OH. Validation of the Malay version of the multidimensional scale of perceived social support (MSPSS-M) among a group of medical students in Faculty of Medicine, university Malaya. Asian J Psychiatry. 2010;3:3-6.

12. Guan NC, Seng LH, Hway Ann AY, Hui KO. Factorial validity and reliability of the Malaysian simplified Chinese version of multidimensional scale of perceived social support (MSPSS-SCV) among a group of university students. Asia-Pac J Public Health. 2015;27:225-31.

13. Nakigudde J, Musisi S, Ehnvall A, Airaksinen E, Agren H. Adaptation of the multidimensional scale of perceived social support in a Ugandan setting. Afr Health Sci. 2009;9(Suppl 1):S35-41.

14. Mohammad AH, Al Sadat N, Yim LS, Chinna K. Validity and reliability of the Hausa version of multidimensional scale of perceived social support index. Iran Red Crescent Med J. 2015;17:0-6.

15. Peplau LA. Loneliness research: basic concepts and findings. In: Sarason I.G., Sarason B.R. (eds) Social Support: Theory, Research and Applications. NATO ASI Series (D: Behavioural and Social Sciences), vol 24. Dordrech: Springer; 1985.

16. Lakey B, Cohen S. Social Support Measurement and Intervention: A Guide for Health and Social Scientists. In S. Cohen, L. G. Underwood, \& B. H. Gottlieb (Eds. ), Social Support and Theory. New York: Oxford University Press. pp. 29-52. https://doi.org/10.1093/med:psych/9780195126709.003.0002

17. Chou K-L. Assessing Chinese adolescents' social support: the multidimensional scale of perceived social support. Personal Individ Differ. 2000;28:299-307.

18. Bagherian-Sararoudi R, Hajian A, Bahrami Ehsan H, Sarafraz MR, Zimet GD. Psychometric properties of the Persian version of the multidimensional scale of perceived social support in Iran. Int J Prev Med. 2013;4:1177-81.

19. Fleury J, Keller C, Perez A. Social support theoretical perspective. Geriatr Nurs N Y N. 2009;30:11-4.

20. Mattson M\& JGH. Linking Health Communication with Social Support. Health Commun. Nexus. 2011.

21. Dahlem N, Zimet G, Walker R. The Multidimensional Scale of Perceived Social Support: a confirmation study. J Clin Psychol. 1991;47:756-61.

22. Zimet GD, Dahlem NW, Zimet SG, Farley GK. The multidimensional scale of Percieved social support. J Pers Assess. 1988;52:30-41.

23. Shrestha SD, Pradhan R, Tran TD, Gualano RC, JRW F. Reliability and validity of the Edinburgh postnatal depression scale (EPDS ) for detecting perinatal common mental disorders ( PCMDs ) among women in low-and lower-middle-income countries : a systematic review. BMC Pregnancy Childbirth. 2016;16:72.

24. Monticone M, Nava C, Leggero V, Rocca B, Salvaderi S, Ferrante S, et al. Measurement properties of translated versions of the Scoliosis Research 
Society-22 patient questionnaire, SRS-22 : a systematic review. Qual Life Res. 2015;24:1981-98.

25. Beaton DE, Bombardier C, Guillemin F, Ferraz MB. Guidelines for the process of cross-cultural adaptation of self-report measures. Spine. 2000;25:3186-91.

26. Stewart RC, Umar E, Tomenson B, Creed F. Validation of the multidimensional scale of perceived social support (MSPSS) and the relationship between social support, intimate partner violence and antenatal depression in Malawi. BMC Psychiatry. 2014;14:1-23.

27. Merhi R, Kazarian SS. Validation of the Arabic translation of the multidimensional scale of perceived social support (Arabic-MSPSS) in a Lebanese community sample. Arab. J Psychiatry. 2012;23:159-68.

28. Ross R, Sawatphanit W, Mizuno M, Takeo K. Depressive symptoms among HIV-positive postpartum women in Thailand. Arch Psychiatr Nurs. 2011;25:36-42.

29. Duru E. Re-examination of the psychometric characteristics of the multidimensional scale of perceived social support among Turkish university students. Soc Behav Personal. 2007;35:443-52.

30. Ramaswamy V, Aroian KJ, Templin T. Adaptation and psychometric evaluation of the multidimensional scale of perceived social support for Arab American adolescents. Am J Community Psychol. 2009;43:49-56.

31. Martins MV, Peterson BD, Almeida V, Costa ME. Measuring perceived social support in Portuguese adults trying to conceive: adaptation and psychometric evaluation of the multidimensional scale of perceived social support. Peritia. 2012;13:5-14.

32. Theofilou P. Translation and cultural adaptation of the multidimensional scale of perceived social support for Greece. Health Psychol Res. 2015;3:9-11.

33. Yang SO, Jeong GH, Kim SJ, Lee SH. Correlates of self-care behaviors among low-income elderly women with hypertension in South Korea. J Obstet Gynecol Neonatal Nurs. 2014;43:97-106.

34. Trujols J, De Diego-Adeliño J, Feliu-Soler A, Iraurgi I, Puigdemont D, Álvarez $E$, et al. The Spanish version of the quick inventory of depressive symptomatology-self-report (QIDS-SR16): a psychometric analysis in a clinical sample. J Affect Disord Elsevier. 2014;169:189-96.

35. Hetherington E, Doktorchik C, Premji SS, McDonald SW, Tough SC, Sauve RS. Preterm birth and social support during pregnancy: a systematic review and meta-analysis. Paediatr Perinat Epidemiol. 2015;29:523-35.

36. Stewart RC, Umar E, Tomenson B, Creed F. A cross-sectional study of antenatal depression and associated factors in Malawi. Arch Womens Ment Health. 2014;17:145-54.

37. Embretson SE, Reise SP. Item Response Theory. Psychology Press; 2013.

38. Khadka J, Huang J, Mollazadegan K, Gao R, Chen H, Zhang S, et al. Translation, cultural adaptation, and Rasch analysis of the visual function (VF-14) questionnaire. Invest Ophthalmol Vis Sci. 2014;55:4413-20.

39. Eker D, Arkar H. Perceived social support: psychometric properties of the MSPSS in normal and pathological groups in a developing country. Soc Psychiatry Psychiatr Epidemiol. 1995;30:121-6.

40. Ersoy MA, Varan A. Reliability and validity of the Turkish version of the internalized stigma of mental illness scale. Turk Psikiyatri Derg. 2007;18:1-7

41. Eker D, Arkar H, Yaldiz H. Generality of support sources and psychometric properties of a scale of perceived social support in Turkey. Soc Psychiatry Psychiatr Epidemiol. 2000;35:228-33.

42. Kuscu MK, Dural U, Önen P, Yaşa Y, Yayla M, Basaran G, et al. The association between individual attachment patterns, the perceived social support, and the psychological well-being of Turkish informal caregivers. Psychooncology. 2009;18:927-35.

43. Nahathai W, Tinakon W. A revised Thai multi-dimensional scale of Percieved social support. Span J Psychol. 2012;15:1503-9.

44. Wongpakaran T, Wongpakaran N, Ruktrakul R. Reliability and validity of the multidimensional scale of perceived social support (MSPSS): Thai version. Clin Pract Epidemiol Ment Health CP EMH. 2011;7:161-6.

45. Saleem M, Tahir MA, UI Huda N. Perceived social support and clinical anger among drug addicts of southern Punjab, Pakistan. Pak J Commer Soc Sci. 2013;7:298-308.

46. Khan MN, Chiumento A, Dherani M, Bristow K, Sikander S, Rahman A. Psychological distress and its associations with past events in pregnant women affected by armed conflict in Swat, Pakistan: a cross sectional study. Confl Health [Internet]. Conflict and Health; 2015;9. Available from: https:// doi.org/10.1186/s13031-015-0063-4

47. Qadir F, Khalid A, Haqqani S, Zill-e-Huma, Medhin G. The association of marital relationship and perceived social support with mental health of women in Pakistan. BMC Public Health 2013;13:1150.
48. Naveed A. Risk factors for postpartum depression, interpersonal relationship anxiety, neuroticism and social support in women with postpartum depression. Pak J Soc Sci. 2015;35:911-24.

49. Akhtar A, Rahman A, Husain M, Chaudhry IB, Duddu V, Husain N. Multidimensional scale of perceived social support: psychometric properties in a south Asian population. J Obstet Gynaecol Res. 2010;36:845-51.

50. Gjersoe NL, Newman GE, Chituc V, Hood B. Individualism and the extendedself: cross-cultural differences in the valuation of authentic objects. PLoS One. 2014;9:e90787.

51. Hamamura T. Are cultures becoming individualistic? A cross-temporal comparison of individualism-collectivism in the United States and Japan. Personal Soc Psychol Rev. 2012;16:3-24.

52. Wild D, Grove A, Martin M, Eremenco S, McElroy S, Verjee-Lorenz A, et al. Principles of good practice for the translation and cultural adaptation process for patient-reported outcomes (PRO) measures: report of the ISPOR task force for translation and cultural adaptation. Value Health J Int Soc Pharmacoeconomics Outcomes Res. 2005;8:94-104.

53. Hardan-Khalil K, Mayo AM. Psychometric properties of the multidimensional scale of perceived social support. Clin Nurse Spec [Internet]. 2015;29:258-61. Available from: https://doi.org/10.1023/A:1005109522457

54. Mokkink LB, Terwee CB, Patrick DL, Alonso J, Stratford PW, Knol DL, et al. The COSMIN study reached international consensus on taxonomy, terminology, and definitions of measurement properties for health-related patient-reported outcomes. J Clin Epidemiol. 2010;63:737-45.

55. Mokkink LB, Terwee CB, Gibbons E, Stratford PW, Alonso J, Patrick DL, et al. Inter-rater agreement and reliability of the COSMIN (COnsensus-based standards for the selection of health status measurement instruments) checklist. BMC Med Res Methodol. 2010;10:82.

56. Prinsen CAC, Mokkink LB, Bouter LM, Alonso J, Patrick DL, de Vet HCW, et al. COSMIN guideline for systematic reviews of patient-reported outcome measures. Qual Life Res [Internet]. Springer International Publishing; 2018;0: 1-11. Available from: https://doi.org/10.1007/s11136-018-1798-3

57. Terwee CB, Mokkink LB, Knol DL, Ostelo RWJG, Bouter LM, De Vet HCW. Rating the methodological quality in systematic reviews of studies on measurement properties: a scoring system for the COSMIN checklist. Qual Life Res. 2012;21:651-7.

58. Terwee CB, Bot SDM, de Boer MR, van der Windt DAWM, Knol DL, Dekker J, et al. Quality criteria were proposed for measurement properties of health status questionnaires. J Clin Epidemiol. 2007:60:34-42.

59. Dambi J, Jelsma J, Mlambo T. A review of the psychometric properties of the cross-cultural translations and adaptations of the Multidimensional Perceived Social Support Scale (MSPSS) [Internet]. PROSPERO Int. Prospect. Regist. Syst. Rev. 2016 [cited 2017 Jan 31]. p. 1-5. Available from: http://www.crd.york.ac.uk/PROSPERO/display_ record.asp?!D=CRD42016052394

60. Dambi JM, Jelsma J, Mlambo T, Chiwaridzo M, Dangarembizi-Munambah N, Corten L. An evaluation of psychometric properties of caregiver burden outcome measures used in caregivers of children with cerebral palsy: a systematic review protocol. Syst rev. Systematic Reviews. 2016;5:42.

61. Dambi JM, Jelsma J, Mlambo T, Chiwaridzo M, Tadyanemhandu C, Chikwanha MT, et al. A critical evaluation of the effectiveness of interventions for improving the well-being of caregivers of children with cerebral palsy: a systematic review protocol. Syst rev. Systematic Reviews. 2016;5:112.

62. Furlan AD, Pennick V, Bombardier C, van Tulder M. 2009 updated method guidelines for systematic reviews in the Cochrane back review group. Spine. 2009;34:1929-41.

63. Norris AE, Aroian KJ, Nickerson DM. Premigration persecution, postmigration stressors and resources, and postmigration mental health: a study of severely traumatized u.S. Arab immigrant women. J Am Psychiatr Nurses Assoc. 2011;17:283-93.

64. Zhou K, Li H, Wei X, Yin J, Liang P, Zhang H, et al. Reliability and validity of the multidimensional scale of perceived social support in Chinese mainland patients with methadone maintenance treatment. Compr Psychiatry. 2015;60:182-8.

65. Cheng ST, Chan ACM. The multidimensional scale of perceived social support: dimensionality and age and gender differences in adolescents. Personal Individ Differ. 2004;37:1359-69.

66. Ling Y, Huebner ES, Liu J, Liu WL, Zhang J, Xiao J. The origins of hope in adolescence: a test of a social-cognitive model. Personal Individ Differ. 2015; 87:307-11.

67. Liu W, Li Z, Ling Y, Cai T. Core self-evaluations and coping styles as mediators between social support and well-being. Personal Individ Differ. 2016;88:35-9. 
68. Yeung A, Slipp LE, Jacquart J, Fava M, Denninger JW, Benson H, et al. The Treatment of Depressed Chinese Americans Using Qigong in a Health Care Setting: A Pilot Study. Evid Based Complement Alternat Med [Internet]. Hindawi Publishing Corporation; 2013;2013:168784. Available from: http:// www.ncbi.n/m.nih.gov/pmc/articles/PMC3639645/.

69. Sing CY, Wong WS. The effect of optimism on depression: the mediating and moderating role of insomnia. J Health Psychol. 2011;16:1251-8.

70. He F, Zhou Q, Zhao Z, Zhang Y, Guan H. Effect of perceived social support and dispositional optimism on the depression of burn patients. J Health Psychol 2016;21:1119-25.

71. Wong SYS, Wong CK, Chan FWK, Chan PKS, Ngai K, Mercer S, et al. Chronic psychosocial stress: does it modulate immunity to the influenza vaccine in Hong Kong Chinese elderly caregivers? Age. 2013;35:1479-93.

72. Li M-Y, Yang Y-L, Liu L, Wang L. Effects of social support, hope and resilience on quality of life among Chinese bladder cancer patients: a crosssectional study. Health Qual Life Outcomes. 2016;14:73.

73. Zeng EQ, Zeng BQ, Tian JL, Du B, Tian XB, Chen H. Perceived social support and its impact on mental fatigue in patients with mild traumatic brain injury. Balk Med J. 2016;33:152-7.

74. Cao W, Li L, Zhou X, Zhou C. Social capital and depression: evidence from urban elderly in China. Aging Ment Health. 2015;19:418-29.

75. Tan J, Ai Y, Wen X, Wu Y, Wang W. Relationship between shyness and loneliness among Chinese adolescents: social support as mediator. Soc Behav Personal Int J. 2016;44:201-8.

76. Liu L, Yang Y-L, Wang Z-Y, Wu H, Wang Y, Wang L. Prevalence and positive correlates of posttraumatic stress disorder symptoms among Chinese patients with hematological malignancies: a cross-sectional study. PLoS One. 2015;10:e0145103.

77. Wang J, Miller JK, Zhao X. Family functioning and social support in men and women diagnosed with depression in China. Contemp Fam Ther. 2014;36:232-41.

78. Wang $Y$, Zhu $X$, Yang $Y, Y i$ J, Tang $L$, He J, et al. What factors are predictive of benefit finding in women treated for non-metastatic breast cancer? A prospective study. Psychooncology. 2015;539:533-9.

79. Chan L. Yu E, li S. Depression in patients receiving peritoneal dialysis. East Asian Arch Psychiatry. 2011:21:99-107.

80. Liu X, Liu C, Tian X, Zou G, Li G, Kong L, et al. Associations of perceived stress, resilience and social support with sleep disturbance among community-dwelling adults. Stress Health. 2015;586:578-86.

81. Zhang B, Ding X, Lu W, Zhao J, Lv Q, Yi Z, et al. Effect of group cognitivebehavioral therapy on the quality of life and social functioning of patients with mild depression. Shanghai Arch Psychiatry. 2016;28:18-27.

82. Chan AWK, Lee A, Suen LKP, Tam WWS. Effectiveness of a tai chi qigong program in promoting health-related quality of life and perceived social support in chronic obstructive pulmonary disease clients. Qual Life Res. 2010;19:653-64.

83. Zhu S, Hu J, Efird JT. Role of social support in cognitive function among elders. J Clin Nurs. 2012:21:2118-25.

84. Taylor-Piliae RE, Haskell WL, Waters CM, Froelicher ES. Change in perceived psychological status following a 12-week tai chi exercise programme. J Adv Nurs. 2006;54:313-29.

85. Vincent-Onabajo GO, Muhammad MM, Ali UM, Masta AM. Influence of sociodemographic and stroke-related factors on availability of social support among Nigerian stroke survivors. Ann Med Health Sci Res. 2015;5:353-7.

86. Mokkink LB, Terwee CB, Knol DL, Stratford PW, Alonso J, Patrick DL, et al. The COSMIN checklist for evaluating the methodological quality of studies on measurement properties: a clarification of its content. BMC Med Res Methodol. 2010;10:22.

87. Park $H$, Nguyen $T$, Park $H$. Validation of multidimensional scale of perceived social support in middle-aged Korean women with diabetes. Asia Pac J Soc Work Dev. 2012;22:202-13.

88. Ng CG, Mohamed S, See MH, Harun F, Dahlui M, Sulaiman AH, et al. Anxiety, depression, perceived social support and quality of life in Malaysian breast cancer patients: a 1-year prospective study. Health Qual Life Outcomes. 2015;13:1-9.

89. Razali SM, Yusoff MZAM. Medication adherence in schizophrenia: a comparison between outpatients and relapse cases. East Asian Arch Pyschiatry. 2014;24:68-74.

90. Ghorbani M, Dolatian M, Shams J, Alavi-Majd H. Anxiety, post-traumatic stress disorder and social supports among parents of premature and full-term infants. Iran Red Crescent Med J. 2014;16:e13461.
91. Roohafza H, Daghaghzadeh AHKH, Afshar H, Zahra Erfani, Adibi P. Life stressors, coping strategies, and social supports in patients with irritable bowel syndrome. Adv Biomed Reseach 2016;5:1-7.

92. Adamczyk K. Development and validation of the polish-language version of the multidimensional scale of perceived social support (MSPSS). Rev Int Psychol Soc. 2013:26:437-66.

93. Adamczyk K, Segrin C. Direct and indirect effects of young adults ' relationship status on life satisfaction through loneliness and perceived social support. Psychol Belg. 2015;55:196-211.

94. Adamczyk K, Segrin C. Perceived social support and mental health among single vs. partnered polish young adults. Curr Psychol. 2015;34:82-96.

95. Adamczyk K, DiTommaso E. Psychometric properties of the polish version of the social and emotional loneliness scale for adults (SELSA-S). Psihol Teme. 2014;23:327-41.

96. Martins MV, Peterson BD, Almeida VM, Costa ME. Direct and indirect effects of perceived social support on womens infertility-related stress. Hum Reprod. 2011;26:2113-21.

97. Martins MV, Peterson BD, Almeida V, Mesquita-Guimarães J, Costa ME. Dyadic dynamics of perceived social support in couples facing infertility. Hum Reprod. 2014;29:83-9.

98. Rey L, Extremera N, Pela'ez-Ferna'ndez MA. Linking social support to psychological distress in the unemployed: the moderating role of Core selfevaluations. Soc Indic Res 2015;127:435-445

99. López-ramos Y. Confirmatory factor analysis for the multidimensional scale of perceived social support in a sample of early retirees enrolled in university programs confirmatory factor analysis for the multidimensional scale of perceived social support in a sample of Ea. Clin Gerontol Routledge. 2016;00:1-12.

100. Guillén Al, Panadero S, Rivas E, Vázquez JJ. Suicide attempts and stressful life events among female victims of intimate partner violence living in poverty in Nicaragua. Scand J Psychol. 2015;56:349-56.

101. Cobb C, Xie D. Structure of the multidimensional scale of perceived social support for undocumented Hispanic immigrants. Hisp J Behav Sci. 2015;37:274-81.

102. Ekback MP, Lindberg M, Benzein E, Arestedt K. Social support: an important factor for quality of life in women with hirsutism. Health Qual Life Outcomes. 2014:12:183.

103. Guan NC, Sulaiman AR, Seng LH, Ann AYH, Wahab S, Pillai SK. Factorial validity and reliability of the Tamil version of multidimensional scale of perceived social support among a group of participants in university malaya medical Centre, Malaysia. Indian J Psychol Med. 2013;35:385-8.

104. Başol G. Validity and reliability of the multidimensional scale of perceived social support-revised, with a Turkish sample. Soc Behav Personal. 2008;36:1303-14.

105. Izquierdo I, Olea J, Abad F. Exploratory factor analysis in validation studies: uses and recommendations. Psicothema. 2014;26:395-400.

106. Zhang G, Preacher KJ. Factor rotation and standard errors in Exporatory factor analysis. J Educ Behav Stat. 2015:40:579-603.

107. Karami $\mathrm{H}$. Exploratory factor analysis as a construct validation tool: (Mis)applications in applied linguistics research. TESOL J. 2016:6:3.

108. Brzyski P, Ko'zka, M, Squires A, Brzostek T. How factor analysis results may change due to country context. J Nurs Scholarsh 2016:48:598-607.

109. Suhr D. Exploratory or confirmatory factor analysis? [Internet]. Stat. Data Anal. 2006 [cited 2017 Jan 28]. p. 1-17. Available from: http://www2.sas.com/ proceedings/sugi31/200-31.pdf

110. Prudon P. Confirmatory Factor Analysis: A Brief Introduction and Critique [Internet]. 2011 [cited 2017 Feb 22]. p. 1-18. Available from: https://pdfs semanticscholar.org/80cc/e29fc22d99c4273bee96ebaa6447c64e268d.pdf

111. Schmitt TA, Sass DA. Rotation criteria and hypothesis testing for exploratory factor analysis: implications for factor pattern loadings and Interfactor correlations. Educ Psychol Meas. 2011:71:95-113.

112. Guo C, Tomson G, Guo J, Li X, Keller C, Söderqvist F. Psychometric evaluation of the mental health continuum-short form (MHC-SF) in Chinese adolescents a methodological study. Health Qual Life Outcomes. 2015;13:198.

113. Drost EA. Validity and reliability in social science research. Educ Res Perspect. 2004;38:105-23.

114. Tavakol M, Dennick R. Making sense of Cronbach's alpha. Int J Med Educ. 2011:2:53-5.

115. Sijtsma K. On the use, the misuse, and the very limited usefulness of cronbach's alpha. Psychometrika. 2009;74:107-20.

116. Beattie M, Murphy DJ, Atherton I, Lauder W. Instruments to measure patient experience of healthcare quality in hospitals: a systematic review. Syst Rev. 2015:4:1-21. 